\title{
Lateralised attentional disruption and resource conservation in persistent
}

\section{depression and anhedonia}

5

6 Anna Bevan ${ }^{1 *}$, Caitlin Hitchcock ${ }^{1}$, Daniel J Mitchell ${ }^{1}$, Tim Dalgleish ${ }^{1,2}$

7

$8 \quad{ }^{1}$ MRC Cognition and Brain Sciences Unit, University of Cambridge, Cambridge, United Kingdom

$9 \quad{ }^{2}$ Cambridgeshire and Peterborough NHS Foundation Trust

10

$11 *$ Corresponding author

12

Email: anna.bevan@mrc-cbu.cam.ac.uk (AB)

13 
Anhedonia - a pervasive loss of interest or enjoyment in relation to previously valued stimuli - is a central feature of clinical depression. A series of studies investigated alterations in perceptual sensitivity and attention, and their relation to anhedonia, in participants with persistent depressive illness in comparison to healthy controls. Study 1 measured perceptual sensitivity in the somatic and auditory modalities while minimising attentional influences. Study 2 maximised attentional influences to assess their impact on perceptual sensitivity in the same modalities. Perceptual sensitivity did not differ between groups in either study. Both studies found evidence of lateralised attentional deficits suggestive of subtle left-sided neglect in the depressed samples. In both studies, lateralised attentional deficits were associated with anhedonia above and beyond depression severity. Study 3 demonstrated through simulation that a single lateralised attentional lapse model could predict the key outcomes in both empirical studies, and additionally demonstrated the plausibility of a resource conservation strategy as a data-generating process among depressed participants. In conclusion, the studies support the novel insight that anhedonia may be related to disruptions in brain networks subserving stimulus-driven attention, with a potential contribution of resource conservation strategies that reduce access to precise sensory information. This finding has implications for clinical practice and may prove to have diagnostic relevance within a precision medicine context.

\section{Introduction}

Major depression is characterised by a central theme of withdrawal or disengagement from the world. Previously valued activities are discontinued, cherished goals may be relinquished and social interactions avoided. Sufferers often report that continued engagement or effort seems pointless and that the savour has gone out of life. Anhedonia - the loss of pleasurable experience in response to previously valued stimuli or activities - is a key feature of depression and may contribute to the sense of meaninglessness that sufferers report. Furthermore, it has been suggested that a dampening or 
numbing of all forms of emotional experience may be typical of depression [1], and perhaps predictive of depression chronicity [2]. At the extreme end of the spectrum of emotional disconnection, there is some evidence that depersonalisation (an altered state of consciousness involving a sense of detachment from the body, emotional experience, willed action or the sense of self) may be associated with chronicity and treatment resistance in clinical depression [3]. A better understanding of the mechanisms underlying anhedonia may provide insight into means of developing more effective and targeted interventions.

It is possible that disruptions in sensory or perceptual experience contribute to the dampening of hedonic or emotional experience in chronic depression [4]. Indeed, there is consistent evidence across modalities that sensory or perceptual processes may be inhibited or suppressed in depressive disorders. In the visual modality, Bubl et al. [5-8] have reported in a series of studies that depression is associated with acute impairments in visual contrast perception. In the auditory modality, one study [9] found reduced sensitivity in auditory stimulus detection during acute depression relative to remission, and a second [10] found similarly affected auditory detection thresholds, particularly on the left side of space, in acutely depressed inpatients relative to healthy controls. A more recent study found evidence of impaired pitch identification in depressed women relative to a control group [11], although it should be noted that the task loaded relatively heavily on memory and the same study found no support for group differences on other indices of auditory perception. In the olfactory and gustatory modalities, several studies suggest that sensory thresholds for detection and discrimination are acutely impaired in depression [12-15] although others have found no support for group differences [e.g., 16, 17].

61 The somatic modality is of particular interest to the question of altered sensory processing in depression, given the likely contribution of specifically somatic or interoceptive signals to emotional 
and hedonic experience [e.g., 18, 19, 20]. The impact of depression on somatic experience has received a great deal of attention, specifically in the context of investigations into pain. Studies examining relative pain thresholds in depression have often included a brief test of perceptual threshold for non-nociceptive somatic stimulation. The results of these tests are conflicting, with some investigators finding reduced sensitivity [21, 22], others finding no differences [23-27] and one finding increased sensitivity [28]. However, to our knowledge there are no studies which constitute a robust investigation of somatic thresholds in clinical depression in a non-stressful context; that is, in which participants are not expecting the imminent infliction of pain.

Therefore, although the evidence is inconclusive, studies exist which suggest that depression may dampen or disturb perceptual experience in every sensory modality. The non-specificity of these findings can most parsimoniously be explained by the hypothesis that apparent group differences in perception are a consequence of the differential functioning of some supra-modal process which acts on all perceptual domains. An obvious candidate for such a process is attention, which, as other commentators have observed [e.g., 4, 9], is difficult to disentangle in its effects from genuine group differences in perception during these tasks. It is well-established that depression can affect attentional processes [29]; in particular, depressed individuals may experience difficulty in sustaining attention over time [30], and in disengaging attention from negative self-referential thinking or rumination in order to attend to external task demands [31]. It is unclear, however, whether depression-related disruptions to attentional processes have any impact on sensory perception.

In summary, it is possible that anhedonia and emotional numbing in persistent forms of depression are related to disturbances of sensory perception. Such disturbances may be modality-specific or arise as a secondary effect of disturbances in a supramodal process such as attention. Two key questions arise from this proposition: first, can genuine differences in perception (for example, in the ability to 
detect sensory signals) be identified independently of attentional factors in persistently depressed individuals relative to healthy controls? And secondly, if attention and perception effects can be differentiated, does attentional dysfunction arising as a result of depression systematically impact on perception? If attentional disturbances in depression can dampen perception in day-to-day life, then it should be possible to model this in the laboratory by showing that relevant attentional manipulations can change the thresholds at which depressed people are able to detect sensory signals. We aimed to evaluate these questions in a series of three studies.

Studies 1 and 2 were experimental, and attempted, respectively, to address questions about the impact of depression on perception independently of attention, and about the impact of depression on perceptual attention and on perception as a secondary consequence. Both studies employed adaptive procedures borrowed from the psychophysics literature which can provide a relatively robust estimate of perceptual sensitivity and which can also generate indices of attentional performance. Both examined perception in two modalities: the somatic modality, selected due to its possible theoretical interest with respect to the issue of anhedonia; and the auditory modality, employed as a control in order to establish whether any group differences generalise at a supramodal level. Study 1 aimed to minimise the impact of attentional factors on task performance to estimate the effect of depression on sensory perception as accurately as possible. Study 2 was designed to replicate and extend the findings of Study 1, but aimed to maximise the impact of attentional factors in order to examine group differences in attention and to assess their impact on perception. Study 3 used a "Bayesian brain" simulation approach to investigate the ability of competing accounts of attentional and perceptual processes to predict the empirical observations made in Studies 1 and 2. 
112 A Bayesian analytic strategy [32-34] was employed in all studies. This approach was chosen because of its flexibility in terms of parameter inclusion and the ease with which it can handle hierarchical and mixture modelling.

\section{Study 1}

117 Study 1 employed a series of psychophysics procedures to estimate perceptual thresholds for somatic and auditory stimuli on the left and right sides of space. Individual perceptual thresholds were estimated using an adaptive staircase paradigm [35], in which participants were asked to make a series of judgements regarding the presence or absence of a stimulus presented at near-threshold intensity.

The aim of the task was to enable comparison of thresholds between depressed and non-depressed groups rather than to identify an absolute threshold for any individual in either modality. In the somatic condition we employed small vibrating motors affixed to the left and right wrists, and in the auditory condition we used external speakers instead of headphones in an effort to render our experimental task as ecologically valid as possible.

Efforts were made to minimise the impact of attentional and response factors that might disproportionately affect depressed participants relative to healthy controls. Specifically, adaptive staircases were used because they can efficiently and reliably estimate thresholds from a relatively small number of trials [36], reducing the potential impact of group differences in the ability to sustain attention. The task was simple, and well-practiced beforehand, reducing load on working memory and executive function, and the testing room was quiet and dark, reducing potential distractions. A twoalternative forced-choice (2AFC) design was employed, requiring a positive response on every trial, to minimise response biases associated with depression [9]. 
Sample characteristics. All depressed participants were diagnosed with chronic, recurrent and/or treatment-resistant forms of depressive illness of at least moderate severity at the time of testing. Mean age of onset was 21.73 years old (SD 11.25) and the mean number of years since first diagnosis was 19.60 (SD 15.89). Six participants (37.5\%) reported that their first onset of depression was in childhood. Six individuals (37.5\%) met criteria for at least one comorbid Axis 1 disorder at the time of testing (five met criteria for one or two anxiety disorders, including Generalised Anxiety Disorder, Obsessive-Compulsive Disorder and Posttraumatic Stress Disorder, and one met criteria for an eating disorder). Six participants (37.5\%) were currently prescribed antidepressant medication ( $n=6$ were prescribed an SSRI, $\mathrm{n}=2$ were additionally prescribed an SNRI). No control participants were prescribed any form of psychotropic medication.

A series of t-tests demonstrated that there were no significant group differences with respect to the characteristics of age, years of education or handedness (see Table 1). As expected, depressed participants differed significantly from healthy controls with respect to self-reported symptoms of depression and anxiety.

Table 1. Study 1 demographic comparisons: Mean (standard deviation) per group, and 2-tailed ttests.

\begin{tabular}{|l|c|c|c|}
\hline & $\begin{array}{c}\text { Depressed group } \\
(n=16)\end{array}$ & $\begin{array}{c}\text { Control group } \\
(n=16)\end{array}$ & \\
\hline \multicolumn{3}{|c|}{ Demographics } \\
\hline Sex & 15 women & 15 women & - \\
\hline Age (years) & $42.00(18.11)$ & $42.50(17.41)$ & $t(30)=0.08, p=0.94$ \\
\hline Handedness (EHI) & $68.14(47.75)$ & $80.06(31.16)$ & $t(30)=0.84, p=0.41$ \\
\hline
\end{tabular}




\begin{tabular}{|l|c|c|c|}
\hline Education (years) & $15.25(2.86)$ & $15.69(2.41)$ & $\mathrm{t}(30)=0.47, \mathrm{p}=0.64$ \\
\hline \multicolumn{3}{|c|}{ Mood measures } \\
\hline Depression (BDI) & $33.19(5.06)$ & $5.06(2.70)$ & $\mathrm{t}(30)=20.77, \mathrm{p}<0.001$ \\
\hline Anxiety (BAI) & $22.00(10.00)$ & $3.06(2.64)$ & $\mathrm{t}\left(17.09^{1}\right)=7.32, \mathrm{p}<0.001$ \\
\hline
\end{tabular}

${ }^{1}$ Degrees of freedom adjusted due to unequal variances

157 GLMM model. A total of 5033 observations were collected, an average of 40 per participant per condition. These data were fit to a generalised linear mixed model with a logistic link function, in which participant responses were pooled across groups in order to estimate logistic regression parameters by group and experimental condition (stimulus modality and laterality) with a random effect added to account for individual differences within groups. Following initial attempts to fit the model (discussed below) a lapse parameter was added which varied by group and condition.

Figure 1 shows the model fit to the data for each group in each condition (left panels), contrasts on the effects on the slope and threshold parameters (upper right panel), and lapse rate estimates by group and condition (lower right panel). As can be seen, there are credible main effects of modality on both threshold and slope, which are unsurprising given differing starting-points and step sizes across the two modalities. There are no other effects on either parameter. Of interest, there are no effects of group, and no interactions between group and any other factor, on either slope or threshold. 

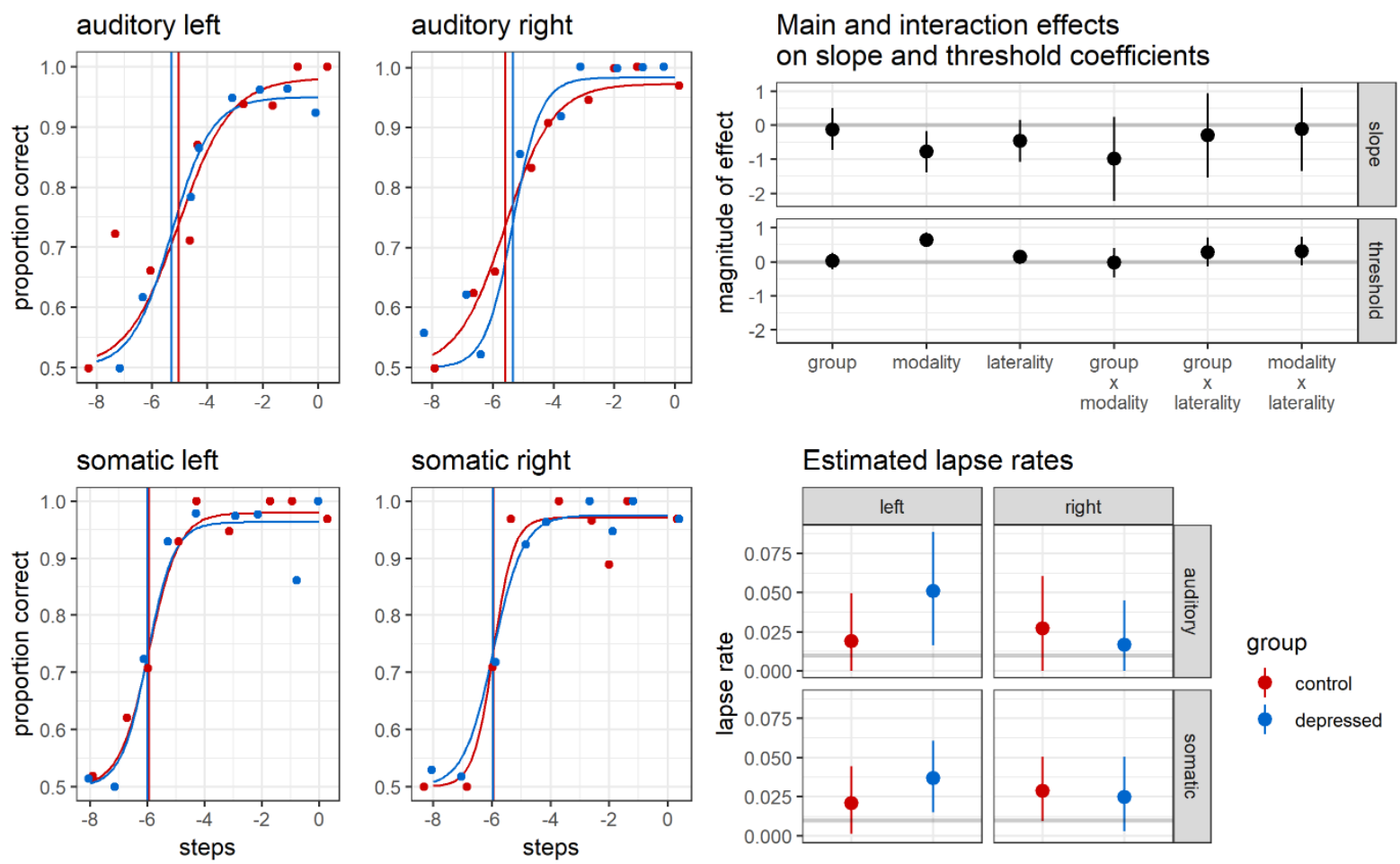

171

172

Figure 1. Study 1 GLMM outcomes. Left panels: mean logistic curves and thresholds (vertical lines) estimated from the Study 1 GLMM model, shown by stimulus modality and laterality. Points represent the observed data (proportion correct averaged over group) shown to the nearest step and jittered to avoid overlap. The step size for the auditory staircases is $4 \mathrm{~dB}$, for the somatic staircases $1 \mathrm{~dB}$. Upper right panel: contrasts on effects and interactions for the slope and threshold parameters. Lower right panel: estimated lapse rates by group and condition.

Lapse rates are estimated on the basis of only a handful of observations, and the estimates shown in Figure 1 are therefore not precise enough to provide credible evidence of any effects of group or condition on the tendency to lapse during the task. Nevertheless, it is noticeable that the only estimates which are credibly larger than zero (using a region of practical equivalence of 0.01 ) are the estimates for left-sided lapse rates among depressed participants in both modalities. 
GLMM model comparison. A variable selection procedure [37] was carried out in order to assess the contribution of the various factors, most importantly group membership, to the fit of the slope and threshold parameters. Eight models were included in the comparison comprising every possible combination of factors. Given the similarity of the effects on the slope and threshold parameters observed in the preceding model, each model tested its effects on both parameters simultaneously. The lapse rate was estimated for all models as above. Models are described in order of decreasing posterior credibility in Table 2.

Table 2. Posterior probabilities and Bayes factors for GLMM variable selection procedure

\begin{tabular}{|c|c|c|c|}
\hline Model & Factors included & $\begin{array}{c}\text { Posterior model } \\
\text { probability }\end{array}$ & $\begin{array}{l}\text { Bayes factor } \\
\text { relative to } \\
\text { reference model }\end{array}$ \\
\hline M & Modality & 0.99997 & $7.56 \times 10^{9}$ \\
\hline ML & Modality and laterality & $2.02 \times 10^{-5}$ & $1.53 \times 10^{5}$ \\
\hline GM & Group and modality & $8.42 \times 10^{-6}$ & $6.37 \times 10^{4}$ \\
\hline REF & None (reference model) & $1.32 \times 10^{-10}$ & 1 \\
\hline$L$ & Laterality & $9.42 \times 10^{-13}$ & $7.13 \times 10^{-3}$ \\
\hline G & Group & $2.59 \times 10^{-13}$ & $1.96 \times 10^{-3}$ \\
\hline GML & Group, modality and laterality & $1.81 \times 10^{-14}$ & $1.37 \times 10^{-4}$ \\
\hline GL & Group and laterality & $2.74 \times 10^{-17}$ & $2.07 \times 10^{-7}$ \\
\hline
\end{tabular}

Model REF is the simplest model comprising a single intercept and slope for both groups of participants in all conditions, and is used here as a reference model. The only models which provide a better account of the data are those which include modality and no more than one additional factor. 
An effect of modality alone is substantially more credible than any other explanation for the data, with the probability of the data under this model increased by a Bayes factor of 49519.76 relative to its nearest competitor.

With respect to hypothesised effects of group, the addition of a group parameter to any model in fact renders it substantially less probable (i.e., $P($ model $G)<P($ model $R E F), P($ model $G M)<P($ model $M)$, $\mathrm{P}($ model $\mathrm{GL})<\mathrm{P}($ model $\mathrm{L})$ and $\mathrm{P}($ model $\mathrm{GML})<\mathrm{P}($ model $\mathrm{ML}))$. This suggests that true group influences on perceptual performance are highly unlikely given the current data, and furthermore that the failure to identify group differences in slope and threshold parameters in the current dataset is not attributable to a lack of power.

Attentional lapses. The lapse rate in a psychophysics task is assumed to be independent of stimulus intensity but is most salient at highly detectable intensities, where errors are unlikely to reflect genuine perceptual limits but can nevertheless exert a disproportionate influence on model fit [38, 39]. A first attempt to fit the model, not including a lapse parameter, resulted in a credible interaction between group and laterality in the slope parameter, observable in both stimulus modalities and reflecting a flattening of the slopes on the left in the depressed group and on the right in healthy controls. This flattening was reminiscent of the influence of unmodelled lapses in psychometric functions [39] and the associated group $\mathrm{x}$ laterality interaction was abolished once the lapse parameter was included in the model. The implication that lapse rates might be disproportionately high on the left in the depressed group and on the right in controls led us to examine asymptotic lapses more carefully.

In order to provide a direct index of attentional lapses, a count of errors occurring at the upper asymptotes was made. For the purposes of this count the upper asymptotes were conservatively 

$0.24, p=0.625$, odds ratio $=0.67)$. in either modality mean. defined as the portion of the curve to the right of the lowest-intensity point at which the percentage of correct responses was greater than or equal to the percentage of correct responses averaged over that data point and all higher-intensity data points. The resulting count of asymptotic lapses for each group in each laterality condition (summed over modality) is shown in Figure 2.
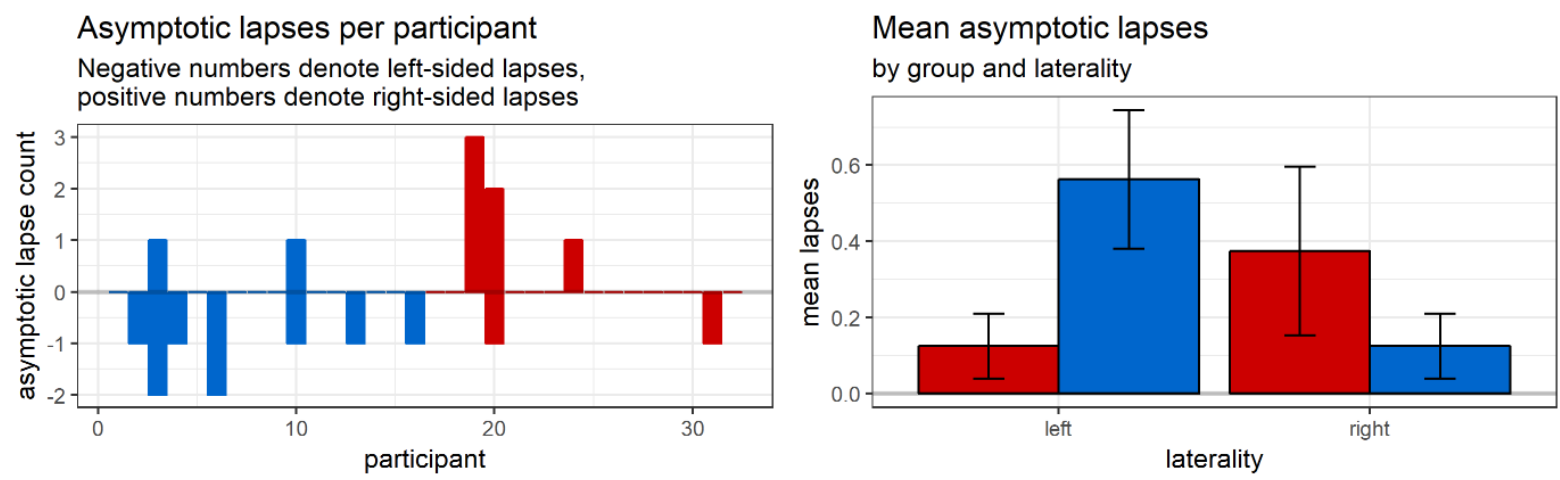

Proportion of group committing at least one lapse

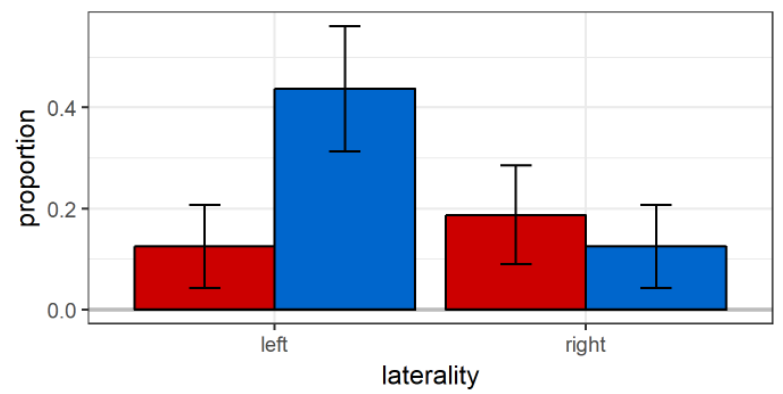

group

control

depressed

Figure 2. Asymptotic lapses by group and participant. Error bars represent 1 standard error of the

Likelihood ratio Chi-square tests confirmed that depressed participants were more than three times as likely as healthy controls to lapse at least once on the left $\left(\chi^{2}(1)=4.04, p=0.044\right.$, odds ratio $\left.=3.5\right)$, while there were no group differences in the likelihood of lapsing at least once on the right $\left(\chi^{2}(1)=\right.$ 
Medication-free analyses. All analyses were repeated excluding $n=6$ participants from the depressed group who were prescribed psychotropic medication at the time of the study. All outcomes of interest were unaffected (Supplementary Materials, Section 1).

\section{Study 1 Discussion}

In summary, under conditions which aimed to minimise any impact of differential attentional resource between groups, and when attentional fluctuations were accounted for, Study 1 provided no evidence for a systematic effect of persistent depression on perceptual threshold in either the somatic or auditory modalities. These findings argue against the existence of modality-specific perceptual disturbances resulting from persistent depression in terms of either somatic or auditory experience. However, they do not rule out the possibility that under conditions of greater attentional load, changes in perceptual sensitivity may arise as a secondary result of a primary disturbance of attention. In line with the hypothesis that depression may have an impact on perceptual attention, there is some indication in the current study of an increased tendency to left-sided attentional lapses in depressed participants, potentially indicating disproportionate difficulty in maintaining attention to the left side of space. Study 2 investigated this possibility further.

\section{Study 2}

In contrast to Study 1 , Study 2 had the explicit aim of maximising the impact of attentional factors which might be expected to moderate perception. Increasing the influence of attentional factors on task performance enables a more detailed examination of depression-specific changes in attention, as well as providing insight into the impact of any such depression-specific attentional changes on perception. In addition, relinquishing the need to keep the task as brief as possible (to minimise attentional lapses or mind-wandering) allowed the collection of a larger number of observations, to enable participant-level analyses. 
The paradigm was similar to that employed in Study 1, with a number of changes designed to increase the 'cost' of performing the task in terms of attentional resources. As before, each participant completed a left-sided staircase and a right-sided staircase for each condition. In contrast to the previous design, however, the left- and right-sided staircases were interleaved and presented in a randomised fashion such that any given trial had an equal probability of belonging to either staircase, and participants decided between spatial (left/right) rather than temporal intervals. This innovation required participants to pay attention simultaneously to both sides of space, increasing the spatial attentional load of the task. Importantly, it also provided the participants with redundant information, allowing them, in principle, to complete the task on the basis of the information provided by only one side of space. As a strategy for identifying "true" perceptual thresholds this is clearly problematic due to the risk of non-converging staircases [see 40 for further discussion of this approach] but in the current context it offers a method of indexing hypothesised lateralised attentional biases. For example, a participant with a strong preference for the right side of space could complete the task by effectively treating the right-sided staircase as a yes-no task, responding "right" with high probability whenever a right-sided stimulus was detected, and "left" with high probability when no stimulus was detected on the right. This would induce a compensatory response bias towards the left at high levels of uncertainty: following an attentional lapse, or when stimulus intensity was low. Given the lateralised effects suggested by Study 1 , it was expected that depressed people would be disproportionately affected by increased spatial load, especially when responding to left-sided stimuli, and that this would be indexed by a compensatory left-sided response bias in depressed participants.

In addition to the increase in spatial load, attentional load was further increased by lengthening timeon-task. Study 2 quadrupled the total number of staircases completed by each participant to sixteen; in addition, each staircase was lengthened by two additional reverses and each block included two 
staircases rather than one, more than doubling the length of time between breaks. The extended timeon-task was expected to impact depressed participants more than healthy controls given the documented effects of depression on sustained attention [e.g., 41, 42].

Competition for attentional resources was further provided by the introduction of depressionrelevant, but task-irrelevant, distractor words of positive, negative and neutral valence. Distraction, and particularly distraction by depression-relevant negative words, was expected to disproportionately impact depressed participants, given the difficulty in disengaging from such material which is associated with the tendency to depressive rumination. Finally, reaction times were recorded in Study 2 as an additional index of attentional control [e.g., 43, 44, 45].

It was hypothesised that an increase in attentional 'cost' would result in a deterioration in overall performance and that this would be exacerbated in depressed individuals. Specifically, it was predicted that increased time-on-task would result in an increase in the likelihood of attentional lapses among depressed participants relative to healthy controls; that increasing the load on spatial attention would induce a left-sided response bias at high levels of uncertainty in depressed individuals; and that the introduction of distractor words would impact differentially on group performance with respect to word category, such that the attentional performance of depressed participants would be more disrupted by depression-relevant negative words relative to healthy controls.

It was an open question whether evidence of distraction or poor attentional control in depressed participants would be associated with a meaningful shift in perceptual thresholds. 
312 Sample characteristics. All depressed participants were diagnosed with chronic or recurrent forms of depressive illness of at least moderate severity at the time of testing. Mean age of onset was 19.25 years old (SD 10.03) and the mean number of years since first diagnosis was 19.67 (SD 14.15). Eight participants (66.7\%) reported that their first onset of depression was in childhood and 11 participants (91.7\%) had suffered too many past depressive episodes to count. Six individuals (50\%) met criteria for at least one comorbid Axis 1 disorder at the time of testing (4 met criteria for an anxiety disorder and 2 met criteria for both an anxiety disorder and an eating disorder). Six participants (50\%) were currently prescribed antidepressant medication. No members of the control group were prescribed any form of psychotropic medication.

A series of t-tests demonstrated that there were no significant group differences with respect to the characteristics of age, years of education or handedness (see Table 3). A maximum-likelihood chisquare test demonstrated that a trend towards a sex difference between groups did not reach statistical significance. As expected, depressed participants differed significantly from healthy controls with respect to self-reported symptoms of depression and anxiety.

Table 3. Study 2 demographic comparisons

\begin{tabular}{|l|c|c|c|}
\hline & $\begin{array}{c}\text { Depressed group } \\
(\mathbf{n = 1 2})\end{array}$ & $\begin{array}{c}\text { Control group } \\
(\mathbf{n = 1 2})\end{array}$ \\
\hline \multicolumn{3}{|c|}{ Demographics } \\
\hline Sex & 10 women & 6 women & $\chi^{2}(1)=3.10, p=0.078$ \\
\hline Age (years) & $38.92(17.36)$ & $33.92(17.53)$ & $t(22)=0.70, p=0.49$ \\
\hline Education (years) & $15.33(2.93)$ & $16.75(2.18)$ & $t(22)=1.34, p=0.19$ \\
\hline Handedness (EHI) & $51.02(74.08)$ & $64.43(57.35)$ & $t(22)=0.50, p=0.63$ \\
\hline
\end{tabular}


${ }^{1,2}$ Degrees of freedom adjusted due to unequal variances.

Hierarchical signal detection model. In total, 20,242 observations were collected (somewhat over

200 per participant per condition). Because of our interest in response bias, the data were fit to a signal detection model $[33,46]$. In this model, indices of perceptual sensitivity (d': the standardised difference between the means of signal and noise distributions) and response bias (c: an index of the marginal probability of giving a particular response) were estimated for each participant in each condition, partially pooling variance through the use of a hierarchical model taking into account individual differences, group membership and the stimulus features of modality and intensity.

Figure 3 shows the model outcomes in terms of the posterior distributions of $d^{\prime}$ and c, (upper and middle panels) and contrasts on the model coefficients (lower panels). The only credible effect on d' is a very strong and expected effect of stimulus intensity. A trend towards an interaction between modality and intensity is not credibly different from zero, and as in Study 1 , there is no indication of any group effect on perceptual sensitivity. On c, there are substantial main effects of group and modality. The group effect reflects a marked tendency towards a left-sided response bias in depressed participants in both stimulus modalities, together with the opposite tendency in healthy controls. The modality effect reflects a tendency among all participants towards right-sided bias in response to auditory stimuli and left-sided bias in response to somatic stimuli. The reasons for this latter finding are unclear, but could include equipment or acoustic imbalances in the testing environment. Trends towards interactions between group and intensity and between modality and intensity are not credibly different from zero. 


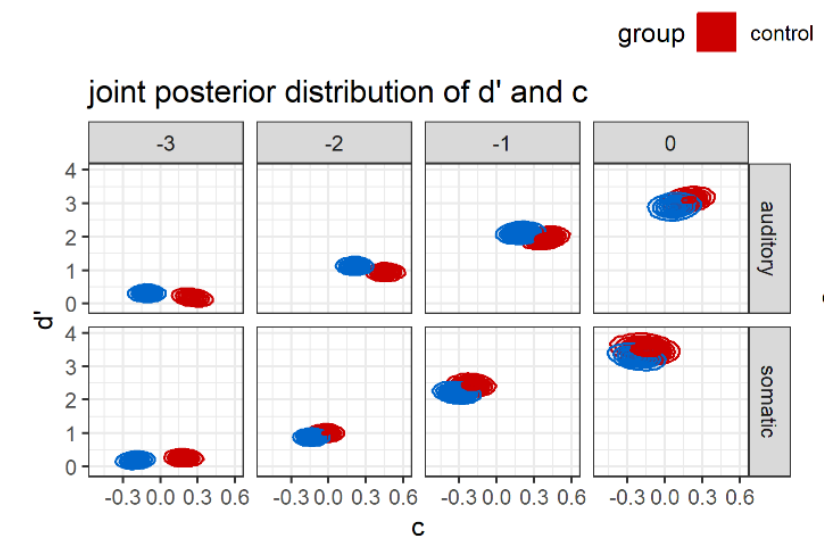

depressed
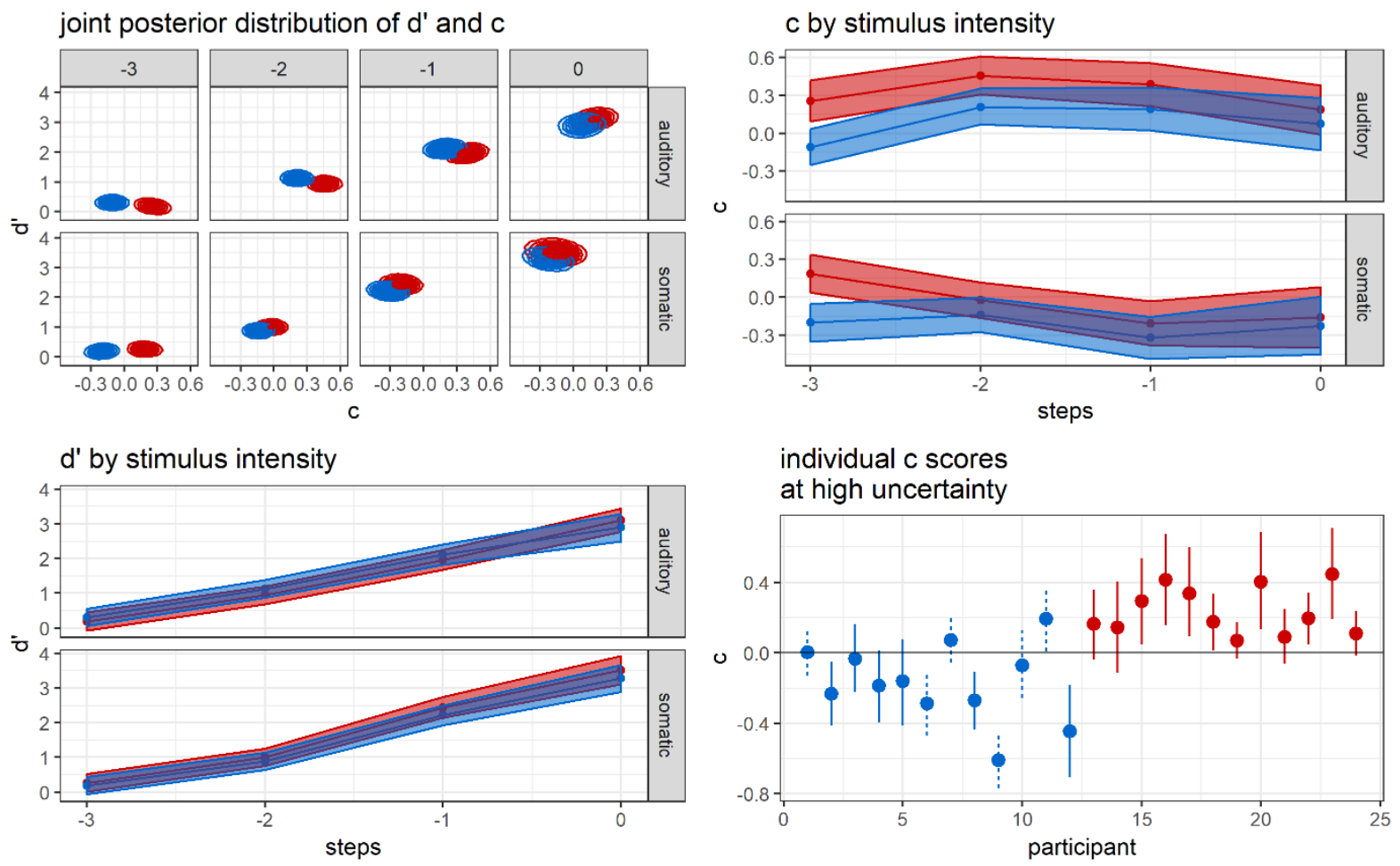

Main and interaction effects on $d^{\prime}$ and $c$
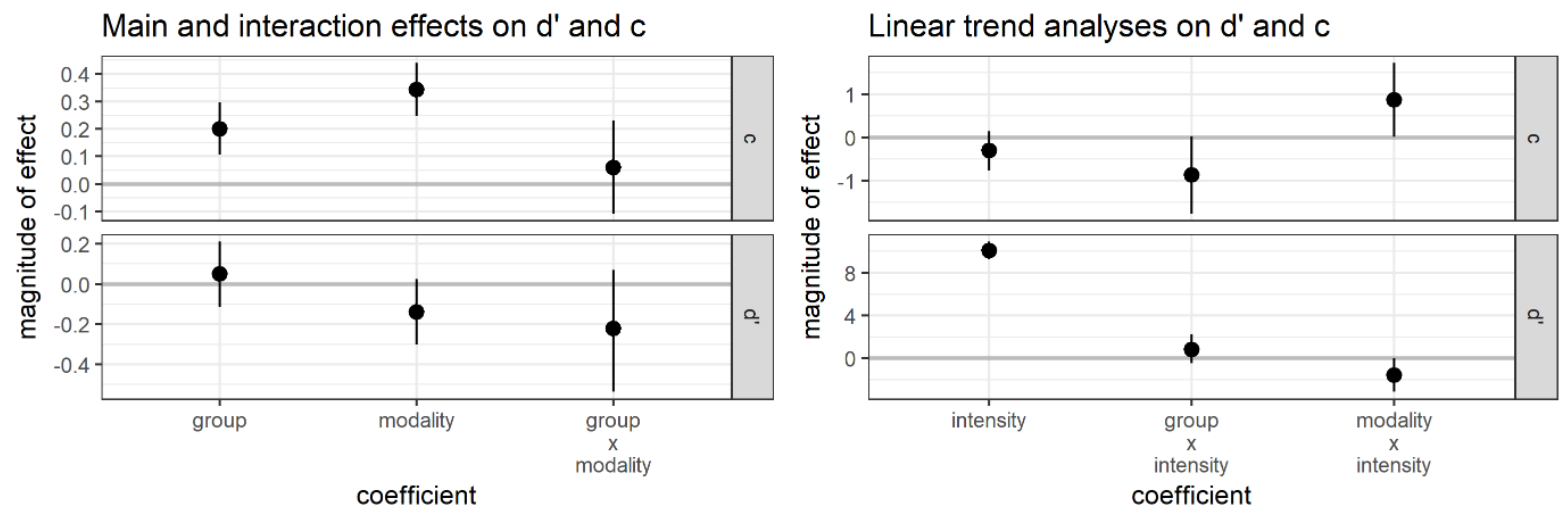

Figure 3. Hierarchical signal detection model outcomes. The top left panel shows the joint posterior distribution of $\mathrm{d}^{\prime}$ and c across stimulus intensity, averaged by group and modality, while the middle left and upper right panels respectively show the corresponding $95 \%$ credible intervals for the averaged estimates of $d^{\prime}$ and $c$ across stimulus intensity. The middle right panel shows the individual participant estimates of $\mathrm{c}$ averaged over modality at the lowest level of stimulus intensity. Unbroken lines indicate that the participant was medication-free. The bottom panels show the main and interaction effects of the linear models on $\mathrm{d}^{\prime}$ and $\mathrm{c}$ : in the bottom left panel simple contrasts on factors 
with 2 levels; in the bottom right panel linear trends on intensity and on the respective interactions of group and modality with intensity. The 3-way interaction (not shown) was not credibly different from zero. Points represent posterior distribution means, error bars $95 \%$ credible intervals.

Signal detection model comparison. A variable selection approach [37] was used to confirm the influence of group on c, and the lack of a group influence on d'. For simplicity, only main effects were included in the model comparison, and only 4 models were compared: a model including an effect of group on both d' and c; a model including an effect of group on d' only; a model including an effect of group on c only; and a model including no effect of group on either parameter. Posterior model probabilities and Bayes Factors are shown in Table 4 and by convention indicate substantial evidence against a group effect on $d^{\prime}$, and strong evidence in favour of a group effect on c [47].

Table 4. Posterior probabilities and Bayes factors for signal detection model comparison.

\begin{tabular}{|c|c|c|c|c|}
\hline \multirow{2}{*}{ Model } & Model & \multicolumn{2}{|c|}{ Posterior model probability } & Bayes factor \\
\hline \multirow{2}{*}{$d^{\prime}$} & & \multicolumn{2}{|c|}{ c } & \\
\hline & Group effect & 0.21 & 0.02 & 10.14 \\
\cline { 2 - 5 } & No group effect & 0.71 & 0.07 & 10.26 \\
\hline Bayes factor & & 0.29 & 0.29 & \\
\hline
\end{tabular}


374 Medication-free analysis. All analyses were repeated excluding $n=6$ participants from the

375

depressed group who were prescribed psychotropic medication at the time of the study. The key outcome of interest (the group difference in response bias) remained highly credible, and in fact its relative likelihood was increased by a factor of 7 by the exclusion of medicated participants. The evidence against a group difference in d' was reduced to an anecdotal level however (Supplementary Materials, Section 1).

Summary. Study 2, like Study 1, found no evidence for an influence of depression on perceptual sensitivity (although the evidence against such an influence was less credible when excluding medicated participants). However, the signal detection model identified a systematic left-sided response bias in depressed individuals which was reversed in healthy controls. In contrast to Study 1 , examination of the data demonstrated no disproportionate tendency towards left-sided asymptotic lapses in the depressed group; however, on the basis of accuracy data alone, it is possible that occasional lapses were masked by the strong response bias.

This hypothesis was investigated using the reaction time (RT) data collected during the Study 2 task. Unusually lengthy reaction latencies are often used as a proxy measure of attentional lapses [e.g., 45], and in line with this approach a distributional analysis was applied to the RT data. It was predicted that an increased tendency to left-sided lapses (represented by unusually lengthy RTs in response to left-sided stimuli) would be observed in the depressed group. 
RT distributions mixture model. The main RT distribution was modelled using a 3-parameter

Weibull distribution following the suggestions of [48]. This distribution is parameterised by a shift parameter $\psi$ which determines the location of the distribution in time, a scale parameter $\theta$ which determines its breadth, and a shape parameter $\beta$ which determines its skew. Following [48], we placed linear models on the shift and (reparameterised) scale parameters $\psi$ and $\theta$, as a preliminary unrestricted random effects model suggested that group differences of interest resided in these parameters but not in the shape parameter $\beta$.

With respect to lapse estimation, [48] suggest that RTs not belonging to the main distribution (i.e., responses which result from fast guesses or attentional lapses) can be best accounted for by expanding the model so that it is a mixture of the Weibull and a pre-specified distribution such as a uniform distribution across the range of RTs. In line with this suggestion, we added a uniform likelihood with upper and lower limits at 0 and 6 seconds to our Weibull model to create a 2component mixture.

Guesses and lapses. A total of 817 RTs (5.32\%) were identified as probable lapses (i.e., were more frequently categorised as belonging to the lapse than to the Weibull distribution). The distribution of these RTs over time was examined by group and laterality (shown in Figure 4: left panel). As can be seen, while the distribution of lapses does not seem to vary depending on stimulus laterality among healthy controls, for the depressed group it appears that fast guesses were more numerous in response to right-sided stimuli, while slower attentional lapses were more numerous in response to left-sided stimuli. 
Distribution of probable lapses and fast guesses by group and laterality

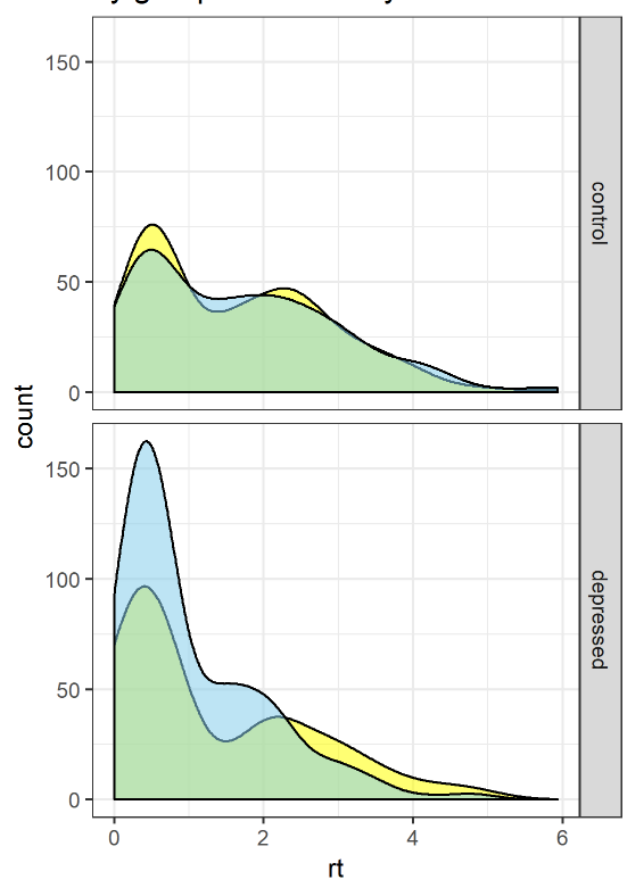

Estimated count of lapses and fast guesses by group and laterality

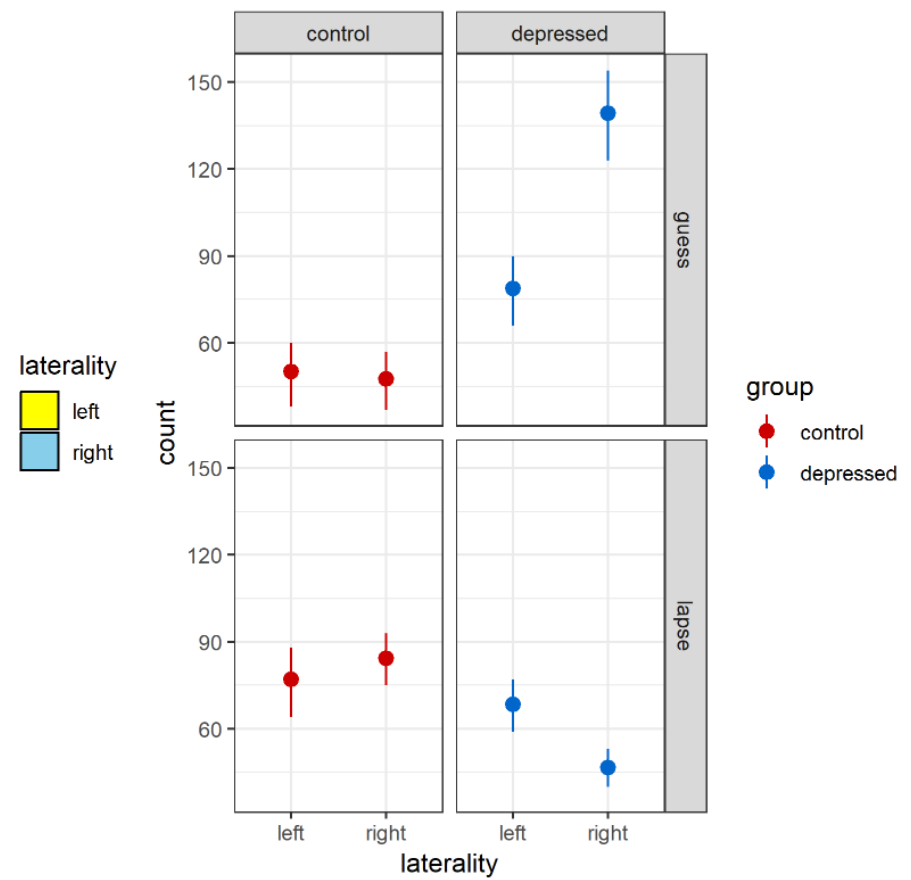

Figure 4. Study 2 estimated distribution and count of fast guesses and attentional lapses as indexed

by reaction time. The left-hand side panel shows the distribution of probable lapses and guesses over time by group and stimulus laterality. The right-hand side panel shows the estimated counts of lapses and guesses once these have been independently captured by separate likelihood distributions. Points represent the posterior distribution mean for each count by group and laterality, and error bars represent $95 \%$ high density intervals.

To distinguish between fast guesses and attentional lapses in a more principled way, an additional pre-specified distribution was added to the mixture model. This distribution was a standard halfnormal likelihood placing high probability over the likely range of fast guesses ( $\approx 0-200 \mathrm{~ms})$ but very little probability over the likely range of attentional lapses ( $\approx 2-6$ seconds).

The posterior distributions of the total count of RTs categorised as guesses and lapses for each group in each laterality condition are shown in Figure 4 (right panel). As expected, lapses among the depressed group are credibly more numerous in trials where the stimulus appeared on the left side of 
434 space compared to trials where it appeared on the right, suggesting, as in Study 1, that depressed

435 participants might have experienced disproportionate difficulty in maintaining attention to the left.

436 Conversely, the depressed group was credibly more likely to respond unusually quickly when the

437 stimulus appeared on the right. The finding of an influence of laterality (i.e., one dependent upon

438 stimulus characteristics) among fast guesses indicates that a substantial proportion of fast responses

439 must have been genuine responses to the stimulus rather than anticipations of it. This finding may

440 suggest an attentional advantage for stimuli occurring on the right side of space, or perhaps a higher

441 level of perceptual or motor preparation for right-sided stimuli relative to left-sided stimuli in

442 depressed participants. Both guesses and lapses arising from left- and right-sided stimuli are

443 comparable among the control group.

445 Although for convenience we refer to RTs categorised as consistent with the respective distributions 446 as "guesses" and "lapses", we do not intend these labels to denote certainty about the causal 447 processes or mechanisms relating to either category, or to imply any assumptions about the underlying distributions. For example, we do not assume that all RTs in one category are drawn from the same underlying true distribution. 
451

452

453

454

455

456

457

458

459

460

461

462

463

464

465

Weibull fits. Key outcomes of the Weibull fit to the bulk of the RT data are shown in Figure 5. As an illustration, Figure 5 (top panels) depicts Weibull distributions arising from the individual posterior parameter estimates averaged by group and over stimulus intensity. These are therefore not fits to actual data, but a visual representation of the effect of average group differences in parameter estimates in terms of the distribution of RTs. The averaged distributions in Figure 5 indicate that the responses of depressed participants are in general faster and more efficiently distributed than those of healthy controls, as discussed below.
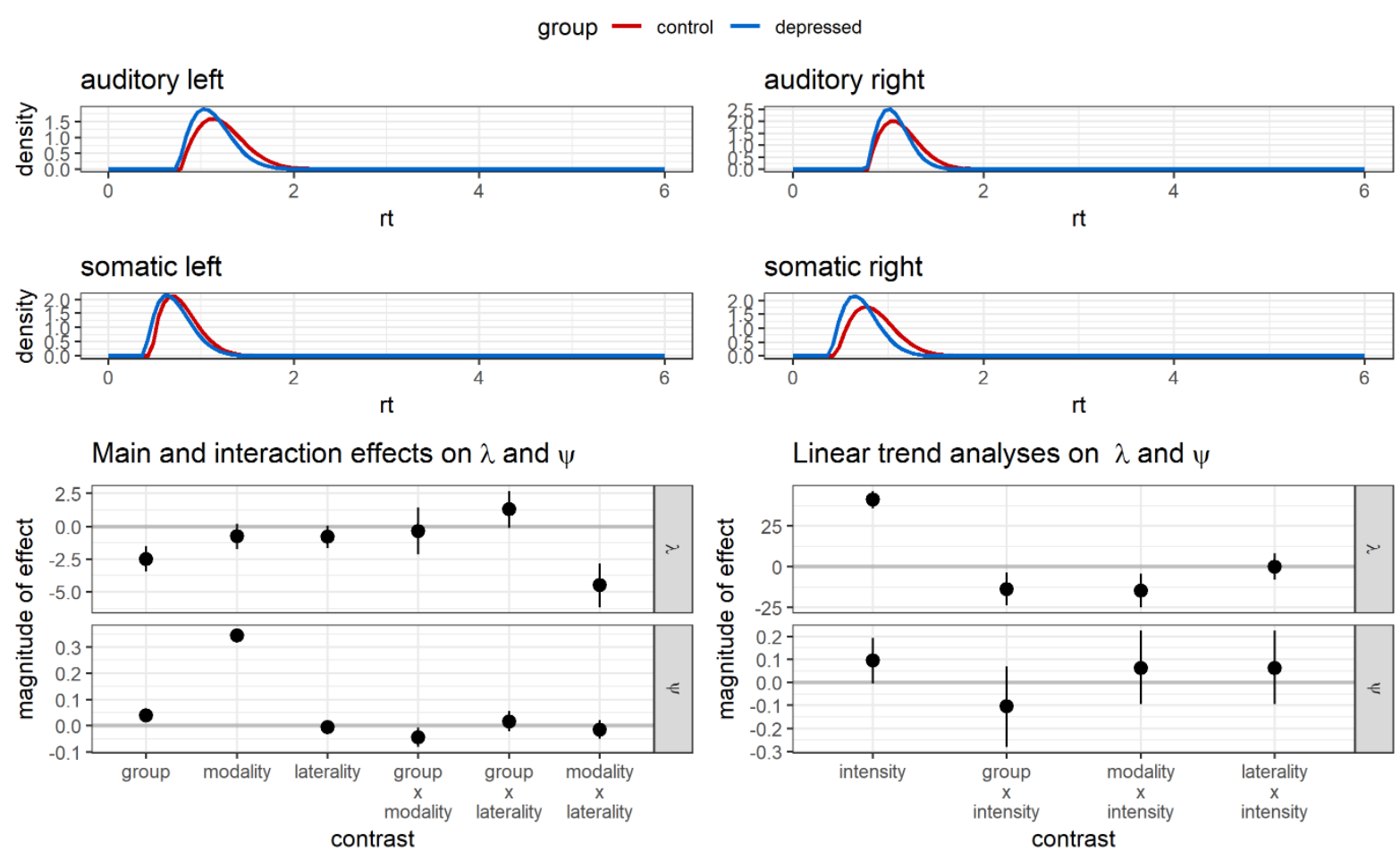

Figure 5. Study 2 Weibull distribution outcomes. The top panels show the most probable Weibull distributions, given the data, averaged over participant and stimulus intensity and shown by group, modality and laterality. The lower panels show the magnitude of the main and interaction effects arising from the linear models on $\lambda$ and $\psi$.

The lower panels of Figure 5 show the main and interaction effects arising from the linear models on the shift parameter $\psi$ and the rate parameter $\lambda$ in the Weibull model. On $\psi$, there was a credible main 
effect of group, indicating rather counter-intuitively that depressed participants demonstrated an ability to prepare and implement a response more quickly than healthy controls. There was also a strong main effect of modality, indicating a delayed lower bound on responses to auditory stimuli relative to somatic stimuli. This effect was not group-specific and may reflect timing differences in the respective perceptual processing systems. A trend towards an advantage for depressed participants when responding to somatic information was not credibly different from zero.

As the rate parameter $\lambda$ increases, the scale parameter $\theta$ decreases. The effects on rate will therefore be discussed in terms of scale, given its greater ease of interpretation in the context of RT distributions. There was a credible effect of group on the scale of the estimated RT distributions, such that the distributions generated by depressed participants were relatively narrow. This, together with the group effect on $\psi$, suggests that RTs in this group were both on average faster and more efficient in terms of reduced RT variability. A credible interaction between group and intensity indicated that the increase in efficiency in the depressed group was particularly marked at higher stimulus intensities.

The remaining effects on $\lambda$ were not group-specific. Most markedly, there was a strong main effect of intensity, such that the spread of RTs was wider when stimulus intensity was lower, consistent with the greater effort or search required to detect lower-intensity stimuli. There were also credible interactions of modality $\mathrm{x}$ intensity (responses to auditory stimuli were relatively efficient at lower stimulus intensities, while the responses to somatic stimuli were relatively efficient at higher stimulus intensities) and modality $\mathrm{x}$ laterality (responses to right-sided auditory stimuli and left-sided somatic stimuli were relatively efficient). These effects are difficult to interpret and may be rather specific to the nature of the stimuli, the hardware used to produce the stimuli, or the acoustic environment of the testing lab. 
491

492

493

494

495

496

497

498

499

500

501

502

503

504

505

506

507

508

509

510

511

512

513

514

515

\section{Study 2 Discussion}

Study 2 aimed to maximise the impact of attentional factors during the perceptual task in order to identify any group differences in attentional performance and to assess their impact, if any, on perceptual sensitivity. Taking the second question first, the findings suggest that even with the increased attentional load of the task in Study 2, there were still no credible overall group differences in perceptual sensitivity, although the marked response biases may suggest that the sensitivity estimates should be treated with some caution. In general, the results suggest that basic perceptual experience (as indexed by sensitivity to perceptual signals) under conditions of top-down attentional focus may in fact be rather robustly preserved in depression, even in the face of attentional challenges including lengthy time on task and the presence of depression-relevant negative distractors. There was also no evidence that stimulus modality interacted with depression status to affect any outcomes with respect to perceptual sensitivity.

Neither the presence nor the valence of task-irrelevant distractor words appeared to disadvantage depressed individuals relative to healthy controls in either attentional or perceptual outcomes. Nor, indeed, did the presence or the valence of distractors impact on attentional or perceptual performance in either group. Conceivably, the distractors were simply not distracting enough: any group differences may have been masked by a floor effect. The outcomes reported here do not allow any further conclusions to be drawn on this topic.

In contrast to the null findings with respect to perceptual sensitivity, Study 2 provides unequivocal evidence of a relationship between depression status and lateralised response biases in perceptual attention. Participant-level analyses demonstrated that neutral or left-sided bias under uncertainty was replicated in $83 \%(n=10)$ of persistently depressed participants - including $100 \%(n=7)$ of those who were medication-free - while right-sided bias was replicated in $100 \%(n=12)$ of healthy controls. 
This is a striking result, despite the small sample size, and unlikely to have occurred by chance. These lateralised response biases were particularly marked when stimulus detectability was low, but may also have existed at high values of $d^{\prime}$ ' when attention wandered, as implied by a disproportionate number of lapses on the left side of space in depressed participants as indexed by the RT distributions. These findings (and the statement 'if I can't hear it, it's on the left' which was independently and spontaneously made by two depressed participants in Study 2) are inconsistent with standard signal detection theory, which assumes that absolute response bias is independent of sensitivity. The relationships between $d^{\prime}$, response bias and group observed here may be more readily interpreted in the context of the indecision model proposed by García-Pérez and Alcalá-Quintana [40], García-Pérez and Alcalá-Quintana [49], García-Pérez and Alcalá-Quintana [50] which incorporates an interval of uncertainty together with a (potentially biased) guessing rule into the standard 2AFC difference model. A guessing rule biased to the left amongst depressed individuals relative to controls can provide a good account of the outcomes observed in the current study as follows: a small proportion of trials at broadly detectable stimulus intensities would nevertheless be expected to yield a d' close to 0 due to lapses of attention or to fluctuations in other variables contributing to its determination. A tendency to guess 'left' on these occasions would result in a mild leftwards bias among depressed participants relative to controls, as observed here at intermediate intensity levels. At the lowest intensity level, where signal is difficult to distinguish from noise, guesses are likely to form a much larger proportion of all responses and the resulting bias would be expected to be commensurately greater. A similarly biased guessing rule, in the opposite direction, can by the same token explain the pattern of results among healthy controls. A biased guessing rule, as suggested above, could also explain the difference between the two studies in terms of the proportion of left-sided asymptotic lapses committed by depressed participants as indexed by accuracy data. A lateralised bias in lapse rates would be expected to be masked by a similarly lateralised bias in guesses. 
541 Assuming that participants employed biased response strategies to maximise the proportion of

542 correct responses, these biases imply a differential level of confidence in the ability to detect a

543 stimulus to one side or the other, assessing one side, perhaps, as disproportionately vulnerable to

544 attentional lapses. In other words, the awareness (conscious or otherwise) that attention is less

545 reliable to one side or the other could explain the use of a corresponding response bias as a

546 compensatory strategy in a motivated participant responding to an undetected stimulus. An

547 alternative explanation assumes that, as well as aiming to complete the task accurately, participants

548 may also have been motivated to conserve attentional resources. In principle it is possible to complete

549 the Study 2 task successfully using information from only one side of space, and the lateralised

550 response bias could conceivably reflect a top-down decision to preferentially allocate attention to one

551 side, with a consequent assumption that undetected stimuli were more likely to occur on the other. If

552 this explanation is correct, control participants chose on average to allocate attentional resources to

553 the left side of space, while depressed participants preferred the right. Following either explanation

554 (response bias as a compensatory strategy, or response bias as part of a strategy for minimising task-

555 related effort), the evident conclusion is that for control participants it is easier or less costly to

556 maintain attention to the left side of space, while for depressed participants it is easier or less costly

557 to maintain attention to the right.

558

559 A related finding arising from the analysis of the main RT distributions indicates that depressed 560 participants systematically responded to stimuli more quickly than healthy controls in terms of both 561 the speed and the efficiency of their responses. This finding is counter-intuitive at first glance and is 562 unlikely on theoretical grounds to reflect a faster speed of processing, more efficient perceptual or 563 motor preparation or increased attentional control in depressed participants relative to healthy 564 controls. However, the theory regarding preferential allocation of attention to one side of space can 565 account for this finding, if it is assumed that depressed participants relied on biased attention to a 
substantially greater extent than did controls. Under these circumstances, one might expect to see a faster and more efficient RT distribution, if only one side of space is carefully monitored; a higher proportion of speeded right-sided RTs, given the attentional advantage for stimuli occurring on the right; and a higher proportion of unusually lengthy left-sided "lapses", given that a left-sided stimulus might be responded to by default only after the participant had satisfied themselves that they had heard nothing on the right.

\section{Study 3}

Study 3 employed a "Bayesian brain" simulation approach to evaluate the plausibility of the interpretations of the data proposed above. A Bayesian "agent" was chosen for its simplicity and intuitiveness, and because Bayesian models of perceptual and attentional processes have demonstrated predictive power in simulating data generating processes in a number of domains [5153]. Previous models have conceptualised perceptual attention as related to the precision of sensory distributions contributing to perception [e.g., 54, 55], and in the current study changes in attention (e.g., attentional lapses) were represented by changes in precision in relevant probability distributions. The simulations aimed to address three questions, as follows:

1) Can a single lapse model, together with a simple compensatory strategy, predict the patterns observed in the data in both studies?

2) Can a "depressed" agent reduce energy expenditure in the Study 2 paradigm by reducing attention to the left side of space without reducing overall perceptual sensitivity as a corollary? If so, what patterns of bias are produced?

3) Are there alternative explanations which can better predict the data? Specifically, how does a constant deficit model or a false percept model compare to the lapse or resource conservation models? 
These questions motivated the development of an agent for each study with similar baseline perceptual sensitivity and behaviour in terms of responses during the task to that observed in study participants. Variants of the two agents (lapsing agents, resource-conserving agents, agents with a constant lateralised attentional deficit and agents prone to false percepts) were then developed in order to address the questions detailed above in turn.

597

\section{Study 3 Results}

Question 1: Can a single lapse model, together with a simple compensatory strategy, predict the Study 2 agents was developed. In both variants, "depressed" agents were defined by a lapse rate of $6 \%$ on the left and $1 \%$ on the right. "Control" agents were defined by a lapse rate of $1 \%$ on the left and $3 \%$ on the right.

604

The spatial nature of the alternatives in the Study 2 2AFC design allowed the Study 2 lapsing agent to compensate for the spatial asymmetry in its attentional performance. Three variants were developed as follows: The lapsing agents were used to generate simulated datasets for each study, and the resulting data were respectively analysed using the GLMM and SDT models employed in Studies 1 and 2. 
616

617

618

619

620

The simulated Study 1 results (shown in Figure 6) closely match those derived from the observed Study 1 data (Figure 1). As with the observed Study 1 data, an attempt to fit a version of the model without any lapse parameter resulted in a credible group $\mathrm{x}$ laterality interaction in the slope parameter which was abolished by the inclusion of a lapse parameter.
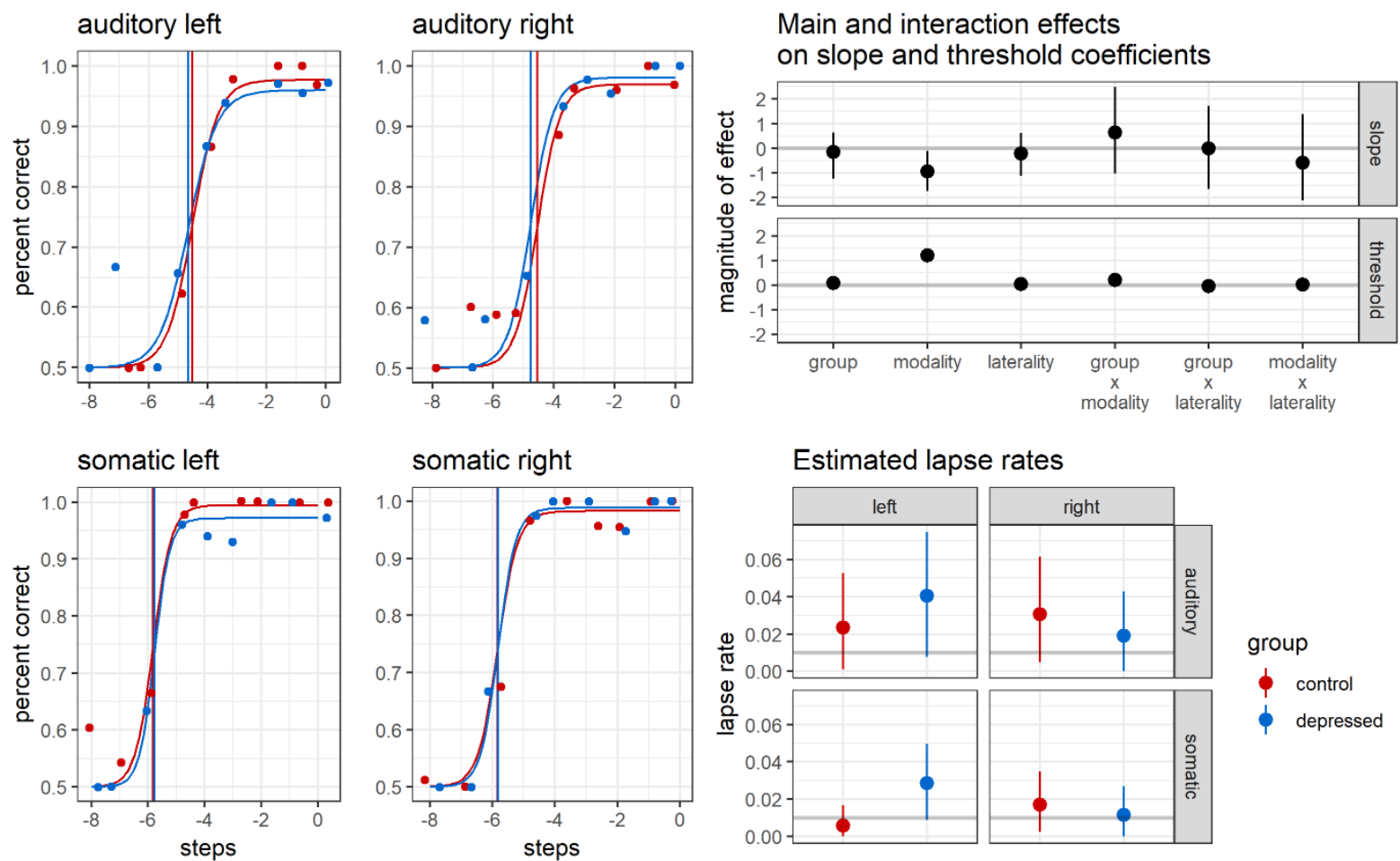

621

Figure 6. Simulated Study 1 GLMM outcomes. Left panels: mean logistic curves and thresholds (vertical lines) estimated from the simulated data fit to the Study 1 GLMM model, shown by stimulus modality and laterality. Points represent the simulated data (proportion correct averaged over group) shown to the nearest step and jittered to avoid overlap. The step size for the auditory staircases is $4 \mathrm{~dB}$, for the somatic staircases $1 \mathrm{~dB}$. Upper right panel: contrasts on effects and interactions for the slope and threshold parameters. Lower right panel: estimated lapse rates by group and condition. Compare to observed data analysis in Figure 1.

The three simulated Study 2 datasets were independently analysed using the Study 2 hierarchical signal detection model, and results are shown in Figure 7 (panels C, D and E). The uncompensated 
lapses variant generated systematic right-sided bias in the depressed agents which was reduced to near zero at the lowest levels of stimulus intensity (Panel C). The intuited lapses variant induced mild left-sided bias in the "depressed" agents, which was broadly constant across all stimulus intensity levels (Panel D). The compensated lapses variant (Panel E), however, closely resembles the observed data (shown in Panel A), with a leftward bias in the "depressed" agents evident across the whole range of stimulus intensity, and particularly marked when stimulus intensity is low.
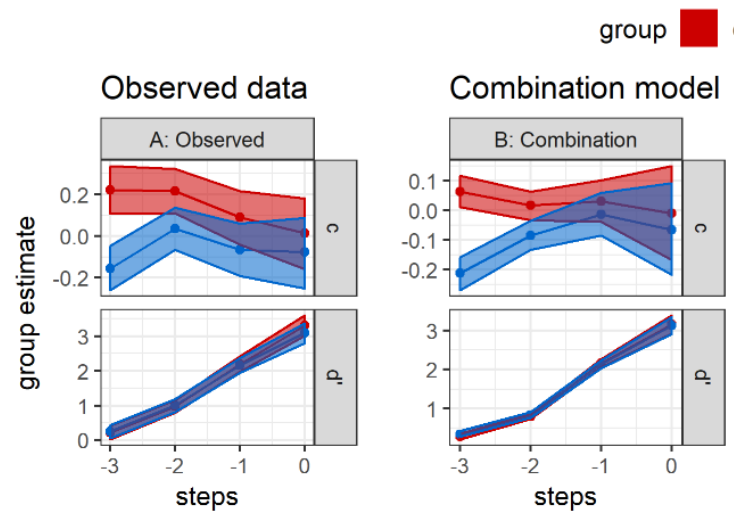
ontrol depressed
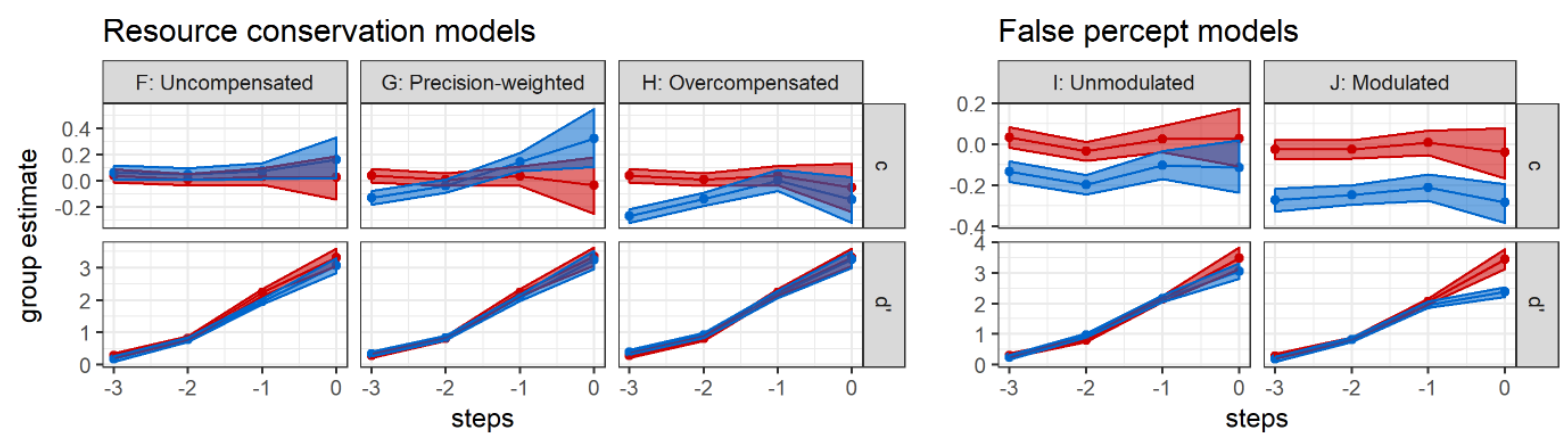

Figure 7. Simulated Study 2 signal detection model outcomes. Posterior distributions of the estimates of $d^{\prime}$ and $c$ by group and stimulus intensity, averaged over modality, for various simulations. Panel A represents the observed Study 2 outcomes for comparison. Points represent the mean posterior estimate for each parameter given group and condition. Ribbons represent the $95 \%$ credible interval of the posterior distributions. Posterior distributions are broader for the observed data than for the simulated data, because for clarity no individual differences were incorporated in the various iterations of the agent in each simulation. 
In summary, simulated data closely resembling the observed data were generated for both Study 1 and Study 2 by an identical lapse model, with the addition in the Study 2 simulation of a simple compensatory strategy: a biased guessing rule. Such a strategy implies that depressed participants are aware at some level of reduced left-sided attentional performance, but does not rely on an ability to accurately intuit lapses. This model appears to provide a good account of the observed data from human participants generated in both studies and is therefore parsimonious as well as internally consistent.

655

\section{Question 2: Can a "depressed" agent reduce energy expenditure in the Study 2 paradigm}

findings that are not addressed by the lapse account include the improved overall RT efficiency and the systematic increase in early responses to right-sided stimuli observed in the depressed group relative to healthy controls. As suggested above, these outcomes could arise from a strategy of directing attention predominantly to the right side of space in order to reduce task-related effort or conserve resources. Arguing against this hypothesis is the fact that no group difference in perceptual sensitivity was found, as might be expected if one group is systematically reducing its access to precise task-relevant information. A second series of modifications were made to the original Study 2 agent to investigate these questions.

A resource-conservation variant of the Study 2 agent was created by attenuating the attention (precision) afforded to the left side of space commensurately with the expectation of the agent that that the left-sided percept would be difficult to detect. Since greater effort is required to detect lowintensity signals, greater cost-savings in energy terms may be made by titrating attention in this way. The resource-conserving agent compensated for its attentional asymmetry as follows: 
1) No compensatory strategy

2) The agent accurately weights evidence from each side of space according to its relative precision

3) The agent uses an overcompensatory precision-weighting strategy

These three variants of the resource-conservation agent were used to generate three simulated Study 2 datasets, which were independently analysed using the Study 2 hierarchical SDT model.

The posterior distributions of the averaged $d^{\prime}$ and $c$ parameters for each variant are shown in Figure 7 (panels $F, G$ and $H$ ). As can be seen in panel F, an uncompensated loss of precision to the left induced a marked right-sided bias which was greatest in response to high-intensity stimuli and reduced to near zero when stimuli were undetectable. As expected, there was also a substantial loss of sensitivity which manifested as a credible group difference in $d^{\prime}$. This group difference in sensitivity was abolished by the compensatory strategy of (accurately) precision-weighting the left- and rightlateralised posterior probabilities (panel G). The pattern of bias arising from this compensatory strategy is midway between the pattern seen in the uncompensated resource conservation simulation and that seen in the observed data. The final variant, employing an over-compensatory precisionweighting strategy (panel $\mathrm{H}$ ), generated data which appears similar to the observed data in terms of group influences on both sensitivity and bias.

In summary, these models critically demonstrate that it is possible to conserve resources by reducing sensory precision to the left when undertaking this task with no loss of apparent sensitivity if response decisions are informed by precision-weighting of the posterior distributions. The pattern of bias arising from this strategy is consistent with the observed data in that left-sided bias increases with increasing uncertainty, but an overcompensatory precision-weighting strategy is required to predict the observed patterns of bias across the whole range of stimulus intensity. 
Summary. The simulations described above indicate that both a lapse account and a resourceconservation account can provide plausible predictions of the observed data. In Study 1, in the absence of any opportunity for resource conservation, the lapse account appears the more probable explanation. In Study 2, both accounts are plausible, and each is consistent with a different aspect of the reaction time data. Perhaps the most likely solution is that a combination of these processes can account for the Study 2 data. Figure 7 (Panel B), for example, depicts averaged outcomes over the compensated lapse model and the overcompensated resource conservation strategy, and displays a similar pattern of sensitivity and bias to the observed data.

707

Question 3: Are there alternative explanations which can better predict the data?

lapse or resource conservation models? In the third section of this study, we searched for competing explanations for our empirical findings. Our first alternative hypothesis, relevant to both studies, suggests that left-sided perceptual performance in depression is affected not by discrete lapses, but by a relatively constant increase in perceptual noise. Our second alternative hypothesis is specific to Study 2, and suggests that left-sided bias was induced in depressed participants not by a compensatory strategy in response to attenuated left-sided attention, but by a predominance of leftsided false percepts, reflecting, on the contrary, heightened attention to the left side of space.

In order to address the first hypothesis, variants of the agents for both studies were created in which the precision of the likelihood on the left side of space was reduced by a small constant rather than a large intermittent value for the "depressed" sample. This reflects a model in which left-sided signals are consistently noisy rather than subject to occasional lapses. Simulated datasets were created on the basis of these variants and analysed as for the lapse variants. 
The outcomes differed substantially from those in the observed data. In the simulated Study 1 dataset

a group by laterality interaction was observed in the threshold rather than the slope parameter, and inclusion of a lapse parameter in the model did not affect the credibility of this interaction. In the simulated Study 2 datasets, loss of sensitivity in an uncompensated version was not corrected by a compensatory strategy of precision-weighting, and patterns of bias did not correspond to those seen in the observed data.

To address the second hypothesis, variants of the "depressed" Study 2 agent were developed with a tendency to false percepts on the left side of space. False percepts may arise when disproportionately precise prior expectations contribute to the generation of disproportionately precise (detectable) percepts despite a small or vague likelihood for the data.

The Study 2 agent was modified to investigate this possibility by increasing the precision of the signal prior on the left side of space while leaving the precision of all other priors and all likelihood functions unchanged. In the first variant, the precision of the left-sided signal prior was increased by a small constant throughout the task. The effect of this was to generate detectable false percepts on the left side of space at higher levels of stimulus intensity, and also to increase confidence in ambiguous leftsided percepts relative to ambiguous right-sided percepts. As a result, sensitivity was markedly reduced at the highest level of stimulus intensity, while a constant pattern of left-sided bias was produced, inconsistent with the observed data (see Figure 7, Panel I). It is possible, however, that the tendency to focus overly on the expectation of a signal is more likely to occur at lower levels of stimulus intensity, when participants may be straining to hear a signal. A second false percept variant was therefore developed which increased the precision of the left-sided expectation of a signal commensurately with the expectation that the right-sided stimulus would be undetectable. The data 
generated by this variant was an even worse match to the observed data (Panel J). It may be the case that a more sophisticated mechanism for updating prior precision would allow the model to predict the data more accurately.

751

In summary, neither of our alternative hypotheses provided as good a prediction of the observed data as the lapse and resource conservation models.

\section{Study 3 Discussion}

Study 3 aimed to investigate possible mechanisms underlying the observed pattern of results from

Studies 1 and 2 using a series of simulations. Although simulations cannot give conclusive insight into the psychological mechanisms underlying the observed results in the experimental studies, they can provide a proof-of-principle with respect to hypotheses about how such mechanisms could theoretically operate. The hypotheses supported by the outcomes of the current study are intrinsically parsimonious by virtue of having been implemented in such a basic Bayesian agent, although of course more complex models could produce equal or better quality of data prediction. Despite its simplicity, the basic agent was able to generate data patterns very similar to those observed in the human data in two different studies by using a single lapse model with a lateralised bias and a proportionally biased guessing rule as a compensatory strategy. This provides some support for the plausibility of the lapse model as a theory about how the observed data were generated. In addition, a resource conservation model importantly demonstrated that the Study 2 task could be successfully completed with no loss of sensitivity by relying predominantly on evidence from the right side of space even when this drastically reduced perceptual precision on the unattended left side of space. This model also succeeded in predicting patterns of bias observed in the data, under conditions where its precisionweighting compensatory strategy was over-compensatory. Both the lapse model and the resource 

availability of task-relevant sensory data. left-sided precision loss or a false percept model. guessing rule.

conservation model provided better predictions of the observed data than either a deficit model of

The simulations provide support for the contention that the observed data may have been generated in part by a compensatory strategy. The pattern of bias in the observed data could not be modelled directly through precision loss or lapses on either side of space because these exerted their smallest effect on bias when stimulus intensity was low. On the other hand, compensatory models utilising biased guess rates or evidence weighting were able to produce a large effect on bias at low stimulus intensity. In fact, accurate compensation did not accurately predict the observed data in either the lapse model or in the resource conservation model. In order to produce left-sided bias across the whole range of stimulus intensity with a larger effect at the lowest level of intensity, it was necessary to overcompensate. This might suggest a relatively broad loss of confidence in the ability to attend to one laterality, or a strategy designed to compensate both for the occasional lapse and for intermittent attention to that side of space. In addition, the simulations provide support for the difference model with guessing proposed by García-Pérez and Alcalá-Quintana [49], given that all simulations which successfully modelled the bias at high uncertainty observed in Study 2 included some form of biased

In summary, the simulations suggest not only that a lateralised attentional deficit is a plausible model for the performance of persistently depressed individuals in these tasks, but also that these individuals may be engaging in heuristic strategies as a means of compensation or resource conservation in order to maintain apparent task performance despite a (voluntary or involuntary) reduction in the 


\section{Anhedonia analyses}

797 The outcomes of all three studies tended towards the conclusion that a lateralised deficit in sensory

798 attention may be present in the context of persistent, moderate-severe depressive illness.

799 Theoretically, however, a question of potentially greater interest is in the relationship between indices

800 of attentional disruption and the phenomenon of anhedonia. This question was investigated by

801 conducting partial correlations between indices of lateralised attentional bias and anhedonia scores

802 derived from the BDI using a subset of items [56] in each of the two studies while controlling for overall

803 depression severity. The best available participant-level estimate of lateralised attentional bias in

804 Study 1 was the absolute count of left-sided asymptotic attentional lapses (see Figure 2), while in

805 Study 2 a more robust index of participant-level attentional bias was provided by maximum a

806 posteriori estimates of response bias under the highest level of uncertainty (as shown in Figure 3).

807 These outcomes were regressed on depression severity (indexed by total BDI-II score) to derive

808 residuals which were then correlated with residuals derived from independent regressions of

809 anhedonia on total BDI score for the two studies respectively. As can be seen in Figure 8 , in both

810 studies there is a credible correlation between indices of left-sided attentional disruption and

811 anhedonia, over and above any contribution of depression severity. A complete description of these

812 analyses is given in the Supplementary Materials (Section 3).

813 


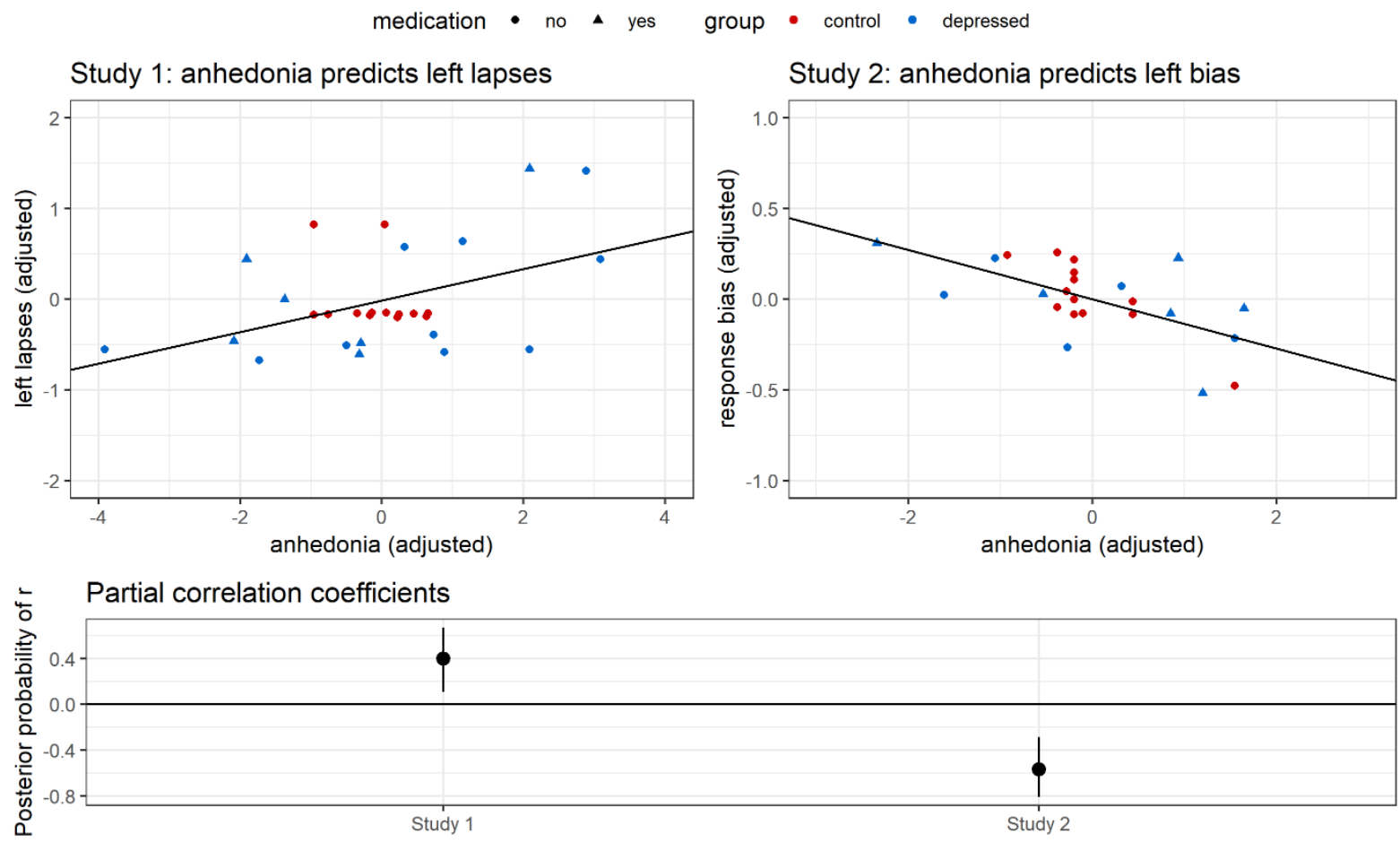

Figure 8. Partial correlations between anhedonia and lateralised attention bias, controlling for

depression severity. Top left: in Study 1, after adjusting for overall depression severity, a higher score

for anhedonia was predictive of a higher rate of left-sided asymptotic lapses. Top right: in Study 2, after adjusting for depression severity, a higher anhedonia score was predictive of an increase in leftsided response bias. Bottom panel: the most probable magnitudes of the two partial correlations are in the region of $0.4-0.6$, while both correlations are credibly different from zero.

\section{General Discussion}

This series of studies investigated the hypothesis that changes in perceptual and attentional experience may be associated with persistent depression, and in particular with anhedonic symptomatology. Study 1 aimed to minimise the impact of attentional factors potentially influencing perception to compare indices of perceptual sensitivity between persistently depressed participants and healthy controls. Study 2 aimed to maximise attentional influences on performance to assess the potential contribution of such influences to shifts in perceptual sensitivity in persistent depression. 
829 Study 3 used a simulation approach to model the empirically-derived outcomes of Studies 1 and 2 to

830 provide proof-of-principle for theories describing possible underlying mechanisms. The results of the

831 studies in terms of both perception and attention were highly consistent with each other.

832

Perceptual sensitivity

834 Study 1 was, to our knowledge, the first study to compare somatic thresholds between depressed 835 participants and healthy controls in a non-nociceptive context. The study's outcomes strongly 836 suggested that once the influence of attentional lapses was taken into account, somatic sensitivity was unaffected by depression status. Similarly, after attention was accounted for, no influence of depression on auditory sensitivity was apparent. Study 2 replicated these findings. However, discernible group-specific effects of attention on perceptual performance were present in all variants of this task in both studies, adding weight to the proposal that attentional processes rather than perceptual sensitivity may have contributed to group differences in previous investigations of somatic and auditory perception [e.g., 9]. Given the relatively small number of participants employed in both studies, and given the possibility that sensitivity performance in depressed participants in Study 2 was inflated by the intelligent use of heuristic compensatory strategies, interpretation must be cautious. However, even the hypothesised compensatory strategies rely on good quality right-sided data for their success, and overall the evidence does not support the theory that depressive symptoms are related to absolute changes in perception in the studied modalities as a result of primary disruption of perceptual processes. Further research is needed into the question of the impact of attentional factors potentially moderating perception in depression. 
851

852

853

854

855

856

857

858

859

860

861

862

863

864

865

866

867

868

869

870

871

872

873

874

\section{Modality}

Neither experimental study provided any evidence that group effects in these tasks were modalityspecific. The laterality effects discussed below were observable in both modalities in both studies, lending support to the theory that they reflect a supramodal process such as attention.

\section{Laterality}

The two experimental studies were consistent in identifying a reliable deficit in attentional outcomes on the left side of space in depressed participants across a range of different attentional indices. This deficit emerged in asymptotic error data in Study 1, in response bias in Study 2 and in the "lapse" component of the mixture model used to analyse the Study 2 RT data. Specifically, both studies found direct evidence for a disproportionate number of lapses on the left side of space in depression, in addition to the more indirect evidence supporting a lateralised lapse model from Study 3 simulations. There was also evidence, although less marked and less consistent, for the opposite tendency towards right-sided attentional lapses and response bias in healthy controls.

As shown by the simulations in Study 3, the observed lateralised response biases are consistent either with a compensatory strategy involving a biased guess rate employed in response to lateralised differentials in confidence in attentional performance; or alternatively, with a top-down strategy intended to conserve resources by enabling task completion whilst allowing attention to be attenuated on one side of space. These possibilities are not mutually exclusive and further research will be required to separate them. In either case, the implication is clear that it is in some way more difficult or more 'costly' for depressed individuals to attend to the left side of space, and, to a lesser extent, more difficult or more costly for healthy controls to attend to the right. 
875 The observed preference of healthy controls for attending to the left side of space is consistent with

876 the well-established phenomenon of pseudoneglect $[57,58]$, thought to reflect right-hemisphere 877 dominance for spatial attention in the majority of the healthy population $[59,60]$. The opposite 878 pattern in depressed individuals is consistent with existing evidence of left-sided deficits in 879 visuospatial and dichotic listening tasks in depressed samples [61-63], and could perhaps be described as presenting similarly to a mild spatial neglect [61]. Spatial neglect as a neurological condition is associated with damage to or disconnection of specific structures in the right hemisphere of the brain, including right-lateralised temporo-parietal junction (TPJ) and ventral frontal cortex $[64,65]$ which have been linked, in healthy individuals, to stimulus-driven aspects of attention [66]. In particular, this 'ventral attentional network', acting in concert with more dorsal regions, is thought to mediate exogenous orienting towards behaviourally relevant stimuli in the environment $[67,68]$; as well as recovery following attentional lapses [45]. The lateralised spatial bias found in the current study, then, may imply not that attention in general (including endogenous or top-down forms of attention) is disrupted in depression and anhedonia, but perhaps that stimulus-driven attentional processes, mediated by right-lateralised ventral areas, may be more specifically affected. It is plausible that disruption to stimulus-driven forms of attention underpinning the ability to orient towards behaviourally relevant stimuli occurring in the external world could contribute to the subjective reduction in meaning or enjoyment in response to previously valued situations or activities seen in anhedonia. However, further investigation is needed to understand these relationships more fully.

In summary, the studies described here found no evidence for a primary deficit in perceptual sensitivity in persistent depression in either the somatic or the auditory modalities. However, both empirical studies demonstrated a reliable, supramodal, group-specific lateralised attentional bias affecting the left side of space in persistently-depressed participants, and the right side of space (consistent with the phenomenon of pseudoneglect) in healthy controls. In addition, in both studies, 
indices of the extent of lateralised bias were associated with anhedonic symptomatology above and beyond their relationship with depression severity. Study 2 response bias and reaction time data additionally suggested that depressed participants might be conserving resources (implicitly or explicitly) by reducing attention to one side of space during the task. Study 3 employed simulations to demonstrate that a simple lateralised lapse model, together with a compensatory strategy employing a biased guessing rule, could generate patterns of data very similar to those observed in both empirical studies. Study 3 also demonstrated the plausibility of a lateralised resource conservation strategy as a data-generating mechanism for the Study 2 data. In conclusion, this series of studies implicates lateralised attentional processes rather than perceptual deficits per se in persistent depression and anhedonia. Further, these findings may indicate a contribution to anhedonia from changes in a rightlateralised ventral attention system which is thought to mediate both pseudoneglect and stimulusdriven attention to behaviourally relevant phenomena in healthy individuals.

\section{Limitations and future research directions}

The studies described here are relatively small and the findings novel. It is encouraging that the key findings from Study 1 were replicated in Study 2, and also that both sets of results could be predicted using the same simple model in Study 3; nevertheless, replication of these effects with larger samples would be valuable before drawing firm conclusions. A related limitation is in the restriction of the depressed group to a single, relatively homogenous population. In order to better understand relationships between attentional disruption and symptom severity, maintenance and vulnerability factors in depression, it will be necessary to generalise the findings to different presentations of depressive illness (for example, by including groups of individuals who are in remission, who present with milder symptomatology, who are experiencing a first episode of depression, or whose depression responds or fails to respond to psychological or pharmacological intervention). If left-sided spatial 
neglect is reliably observable in individuals with persistent or treatment-refractory forms of depression, this is potentially valuable as a diagnostic tool in a precision medicine context.

Future work employing alternative psychophysics paradigms (for example, a method of constant stimuli approach) could enable a more comprehensive analysis of relevant psychometric functions in depression, while attentional fluctuations could be estimated more elegantly through the use of strategies intended to assess non-stationarity in psychometric procedures [e.g., 69, 70]. A particularly interesting approach might involve comparing the psychometric profile of an individual during an episode of major depression with the psychometric profile of the same individual in remission or recovery. In terms of intervention development, examination of changes in attention in response to a wider range of stimuli and contexts could also be of interest, particularly with respect to study designs which can more directly identify resource conservation or the use of heuristic strategies in depression and their impact on perceptual or hedonic experience.

Further work is also needed to investigate the neural mechanisms of the relationship between lateralised attentional disruption and anhedonia identified in the current series of studies. In particular, the large and group-specific effects of stimulus laterality on attentional performance suggest that future investigation of right-lateralised brain networks mediating stimulus-driven attention may be a fruitful avenue for furthering our understanding of persistent forms of depression and of anhedonic symptomatology in particular.

Finally, the key motivation for this work was the imperative to develop new and more effective psychological treatment approaches for depressed individuals experiencing chronic, recurrent and treatment-resistant illness episodes. Sensory attention may be a particularly worthwhile object of study in terms of mechanisms potentially driving persistent depression and anhedonia, because it has 
the advantage of being consciously modifiable and is thus a potential target for psychological treatment. Indeed, interventions such as Mindfulness-Based Cognitive Therapy [71], with a substantial focus on the training of sensory attention, have already proved effective in the acute [72] or prophylactic [e.g., 73] treatment of more persistent forms of depression. The "active ingredients" which contribute to the effectiveness of $\mathrm{MBCT}$ are a matter of debate at present: it is very possible that our findings have relevance for this question. The relationships demonstrated here between disruption in lateralised attention, depression and anhedonia may also suggest new avenues for the development or elaboration of similarly attention-focused approaches in psychological therapies.

\section{Materials and Methods}

For each of the two experimental studies, an independent sample of moderately-severely depressed individuals with histories of chronic, treatment-resistant or recurrent depressive illness was recruited. Each sample was matched to an equal number of healthy controls on age, sex, handedness and years of education. Participants were recruited from an established panel of potential research volunteers. Major Depressive Disorder (MDD) was diagnosed via clinical screening assessments including a Structured Clinical Interview for DSM-IV Axis I disorders [SCID-I: 74] administered by a trained research assistant, and a SCID mood module administered on the same day as testing by a registered clinical psychologist. Depression and anxiety severity were estimated using the Beck Depression Inventory [BDI-II: 75] and the Beck Anxiety Inventory [BAI: 76] respectively. Inclusion criteria for the depressed groups were a diagnosis of either chronic or recurrent MDD in the context of a current Major Depressive Episode of at least moderate severity, defined as scoring 20 or above on the BDI-II [77].

971 Exclusion criteria included current alcohol or substance dependence or a history of psychosis, bipolar 972 disorder, traumatic brain injury, intellectual disability or dementia. Exclusion criteria were similar for the control groups, with the additional specification that controls had no significant history of anxiety 
or depression, and scored within the 'minimal' range on both self-report measures at the time of testing: i.e., a score of less than 14 on the BDI-II [77] and a score of less than 8 on the BAI [78].

976

977 All participants provided written informed consent before taking part, and received compensation of f6 per hour for their participation. The studies were conducted in accordance with ethical approval from the Cambridge Psychology Research Ethics Committee (PRE.2013.72).

980

\section{$981 \quad$ Materials}

Somatic stimulation was delivered via one of two $10 \mathrm{~mm}$ diameter vibrating tactors (310-103, Precision Microdrives) affixed to the participant's left and right ventral forearms using surgical tape (see Figure $9 \mathrm{~B}$ for the layout of the experimental materials). The tactors were driven using an Arduino board (Arduino Uno, Arduino) connected to a desktop PC via a USB port and controlled via Matlab (2009a, White noise $(\mathrm{LAeq}=65 \mathrm{~dB}$ ) was continuously delivered via external speakers (described below) during somatic blocks to mask any sounds generated by the tactors when driven. 
A

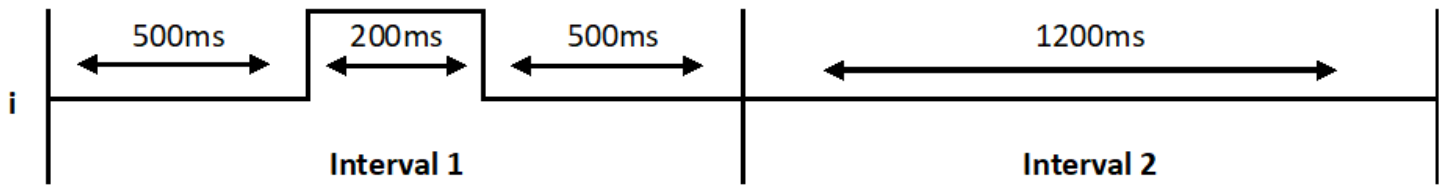

ii

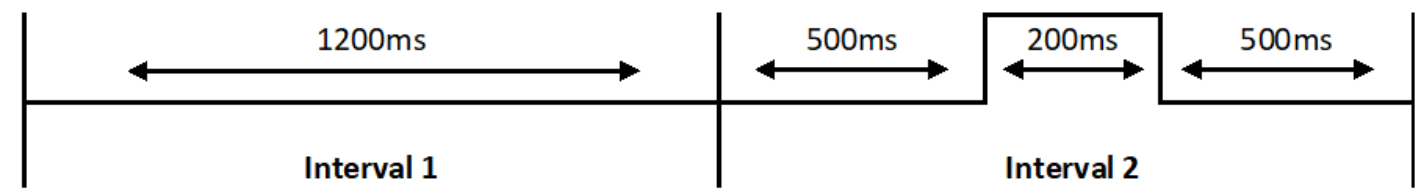

B

990
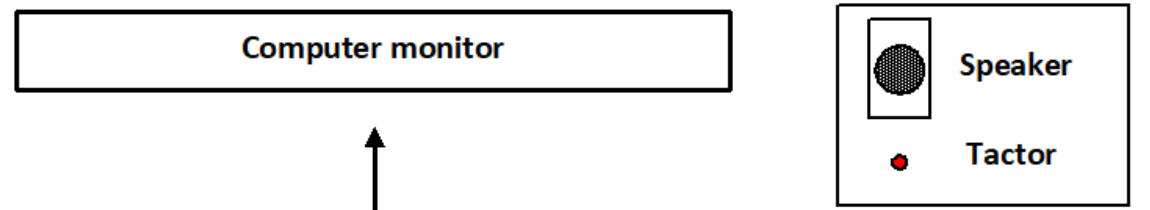

$500 \mathrm{~mm}$
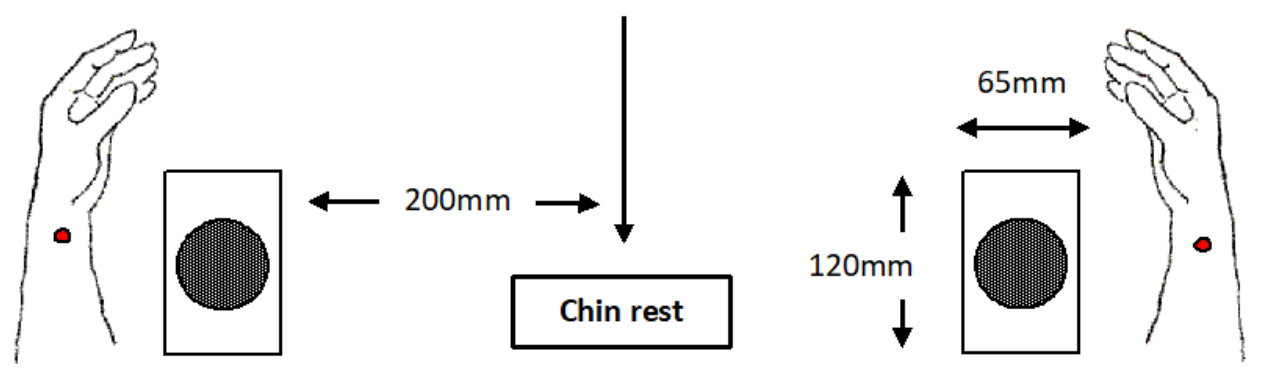

Figure 9. Experimental materials. A: a trial in Study 1. The stimulus is presented in either Interval 1 (i) or Interval 2 (ii). Sequence $\mathbf{i}$ or ii is randomly selected. B: Participants were seated at a table facing a computer monitor with forearms resting on small pads affixed to the table and head steadied by a chinrest. Tactors were affixed to the participant's left and right ventral forearms using surgical tape. Foot pedals were adjusted to a comfortable position under the table. Intervals were indicated by the appearance of the relevant interval name in the centre of the computer screen for the $1200 \mathrm{~ms}$ duration of each interval. Participants fixated on a cross in the centre of the screen between trials. Lights were dimmed during the task. 
1000

1001

1002

1003

1004

1005

1006

1007

1008

1009

1010

1011

1012

1013

1014

1015

1016

1017

1018

1019

1020

1021

1022

Auditory stimulation was delivered via one of two small mylar speakers $(50 \mathrm{~mm}$ diameter) fixed to the left and right of the participant (see Figure 9B). The speakers were connected to a soundcard incorporated into a desktop PC running Matlab (2009a, The Mathworks). Stimuli consisted of $440 \mathrm{~Hz}$ pure tones lasting $200 \mathrm{~ms}$, delivered via Matlab and the psychophysics toolbox [79, 80]. All aspects of the stimuli were kept constant throughout the experiment with the exception of their intensity.

Given that finger movements can generate somatic sensations in the wrist area, responses during all blocks were made using foot pedals for left and right feet, to avoid the possibility of interference with somatic detection on the wrist.

Study 2 word lists. Distractor words were drawn from three lists consisting respectively of 82 depression-relevant negative words, 82 positive antonyms, and 82 neutral words (Supplementary Materials, Section 2), all selected from the stimulus set compiled by Warriner, Kuperman [81]. Oneway ANOVAs demonstrated that word lists were adequately matched on word length and frequency. Independent samples t-tests confirmed that negative words were both more negative in valence $(t(162)=24.22, p<0.001, d=1.77)$ and more arousing $(t(162)=7.86, p<0.001, d=1.27)$ than neutral words, while positive words were both more positive in valence $(t(162)=18.63, p<0.001, d=1.64)$ and more arousing $(t(141.54)=6.47, p<0.001, d=0.90)$ than neutral words. Affective ratings differed significantly between negative and positive words ( $t(162=40.72, p<0.001, d=1.90)$ but arousal ratings did not $(\mathrm{t}(146.42)=0.08, \mathrm{p}=0.94, \mathrm{~d}=0.01)$. Welch's $\mathrm{t}$-tests correcting for unequal variances were used for the positive-neutral and negative-positive arousal comparisons.

\section{Procedure}

Study 1. Participants were verbally requested to pay attention to either the left or right side of space (depending on block) while a series of stimuli were presented. Stimulus intensity was varied according 
to a standard psychophysical 3-down 1-up adaptive staircase procedure [35], where the accuracy of the previous series of responses determined increases or decreases in intensity until 8 reverses had been completed. This procedure converges on a threshold intensity for each participant at which approximately $79 \%$ of stimuli are detected. Initial step sizes were $8 \mathrm{~dB}$ for auditory stimulation and $2 \mathrm{~dB}$ for somatic stimulation, reducing by an eighth with each reverse in order to increase the precision of the final estimate. Initial stimulus intensities were well above normal perceptual thresholds $(0.9 \mathrm{G}$ for the somatic stimulus and $55 \mathrm{~dB}$ LAeq for the auditory stimulus) and a 1-up-1-down procedure was used until the first reverse in order to reduce the time taken to reach the proximity of each participant's threshold.

A 2AFC paradigm was used - requiring a positive response on each trial - to reduce the likelihood that individual differences in motivation or mood could introduce a systematic response bias between groups. Stimuli were presented during one of two randomly selected time intervals (illustrated in Figure 9A). Intervals were indicated by a visual signal on the computer screen ("Interval 1" at fixation for the duration of the first interval, and "Interval 2" for the duration of the second, followed by a question mark which remained visible until a response was made). Accurate responses were a left foot-press for a stimulus occurring in the first interval, and a right foot-press for a stimulus occurring in the second. Responses made prior to the appearance of the question mark were not recorded, and following a valid response there was an interval of $1500 \mathrm{~ms}$ before the next trial began. Four blocks were presented in total: somatic left, somatic right, auditory left, and auditory right. Block order was counterbalanced across participants using a Latin Square. Participants were informed that one stimulus would occur in each trial, with a $50 \%$ chance of occurring in either interval.

Stimuli were demonstrated to participants before starting the first block, and an opportunity was given to practice depressing the footpedals. 
Study 2. Staircase step sizes were made constant ( $1 \mathrm{~dB}$ in the somatic modality, $4 \mathrm{~dB}$ in the auditory modality) for ease of analysis. Starting intensities were initially reduced to reflect this change (leadins would otherwise be doubled in length) but were varied where necessary in the course of testing to ensure that all participants began the staircases at an intensity that they could comfortably detect. Staircases were lengthened from 8 to 10 reverses to provide more data and to increase time on task.

Each participant completed interleaved left- and right-sided staircases for each condition, making a judgement as to whether the stimulus in each trial had occurred on the left or the right side of space. Participants were alerted to the beginning of a trial by the appearance of either a fixation cross or an irrelevant word on the screen. After $500 \mathrm{~ms}$, a stimulus was presented either on the left or the right, and participants responded using the spatially congruent footpedal. Once a response had been made, the trial indicator vanished from the screen and a $1000 \mathrm{~ms}$ ITI intervened before the beginning of the next trial. Response latencies were recorded by the program.

1064

Each participant completed four blocks in each modality (each block consisting of two interleaved staircases as described above). During the first block of each modality, the trial indicator consisted of a fixation cross. In the three subsequent blocks (presented in counterbalanced order across participants), the indicator consisted of a word drawn at random (with replacement) from one of the three lists described above, depending on block.

Two brief practice blocks (one for each modality) were completed by each participant prior to beginning the task. The function of the practice blocks was to ascertain that the participant had understood the task and was able to utilise the foot-pedals comfortably. No feedback was given. 


\section{Models}

1076

1077

1078

1079

1080

1081

1082

1083

1084

1085

1086

1087

1088

1089

1090

1091

1092

1093

1094

1095

\section{Statistical models}

Statistical models were fit using an Markov Chain Monte Carlo (MCMC) approach, conducted using JAGS [82], running with $\mathrm{R}$ [R Core 83] and the runjags [84] and coda [85] packages. For each parameter estimate within each model, four chains were initialised at a range of starting points and run until convergence, assessed visually and according to the potential scale reduction factor ( $\hat{\mathrm{R}}:$ Gelman \& Rubin, 1992). Once adequate convergence was identified, chains were subsequently run until an estimated sample size near or above 10,000 samples was collected for each parameter of interest [34]. Plots were produced using the packages ggplot2 [87] and ggpubr [88].

GLMM model (Study 1). According to the model, the data are distributed as

$$
y_{i j k} \sim \operatorname{Binomial}\left(n_{j k}, \theta_{j k}\right)
$$

where $y_{\mathrm{ijk}}$ denotes the $\mathrm{i}^{\text {th }}$ observation for the $\mathrm{j}^{\text {th }}$ participant in the $\mathrm{k}^{\text {th }}$ condition, $\mathrm{n}_{\mathrm{jk}}$ denotes the total number of observations and $\theta_{\mathrm{jk}}$ the rate of correct responses of participant $\mathrm{j}$ under condition $\mathrm{k} . \mathrm{K}$ is equivalent to a design matrix including a group factor ( 2 levels), a modality factor ( 2 levels), and a laterality factor (2 levels).

Rate of correct responses $\theta_{\mathrm{jk}}$ was modelled as a logistic transform of a combination of intercept and slope varying by condition $\mathrm{k}$ and a random factor $\mathrm{Rj}$ specific to the participant. Because the task was a $2 \mathrm{AFC}$ task, the lower asymptote was set at the guess rate, $\gamma=0.5$. Following initial attempts to fit the model (discussed above) a lapse parameter $\lambda$ was also added.

$$
\theta_{j k}=\gamma+\left(1-\gamma-\lambda_{k}\right) \operatorname{logistic}\left(\beta 0_{k}+\beta 1_{k} x+R_{j}\right)
$$

Lapse rates $(\lambda)$ were modelled using a uniform prior [39] with a relatively permissive upper bound of $10 \%$ given that our participants were drawn from a clinical population. Lapse rates were allowed to vary by group and condition. 


$$
\lambda_{k} \sim \text { Uniform }(0,0.1)
$$
data:

$$
\begin{gathered}
\beta 0_{k} \sim N\left(0, \sigma^{\beta 0^{2}}\right) \\
\beta 1_{k} \sim N\left(0, \sigma^{\beta 1^{2}}\right) \\
R_{j} \sim N\left(0, \sigma^{R^{2}}\right) \\
\sigma^{R}=\sqrt{\frac{1}{\tau^{R}}} \\
\tau^{R} \sim \operatorname{Gamma}(0.001,0.001) \\
\sigma^{\beta 0}=\sigma^{\beta 1}=2
\end{gathered}
$$

1100

1101

1102

1103

1104

The predictor variable was binned into 8 steps, each step representing $4 \mathrm{~dB}$ in the auditory staircases and $1 \mathrm{~dB}$ in the somatic staircases; and standardised using z-score scaling.

Hierarchical SDT model (Study 2). Within each modality, data were pooled across blocks following preliminary analyses indicating no effect of distractor presence or valence on perceptual thresholds in either modality. Observations near the upper and lower asymptotes were binned in order to render estimates at the extremes more reliable. Specifically, participants performed at chance at $-12 \mathrm{~dB}$ in the auditory condition and $-6 \mathrm{~dB}$ in the somatic condition and observations occurring at these and lower intensities were therefore pooled together in one category. Similarly, participants in the somatic condition performed at ceiling at $0,-1$ and $-2 \mathrm{~dB}$, and therefore these observations were pooled. Any observations occurring above the starting intensity were included in the starting intensity category in both modalities. This produced 4 levels of stimulus intensity in each modality.

Arbitrarily, hits were defined as correct left-sided responses while correct rejections were defined as correct right-sided responses. 
According to the model, the data are distributed as

$$
\begin{aligned}
& h_{j k} \sim \operatorname{Binomial}\left(n_{j k}^{h}, \theta_{j k}^{h}\right) \\
& f_{j k} \sim \operatorname{Binomial}\left(n_{j k}^{f}, \theta_{j k}^{f}\right)
\end{aligned}
$$

where $h_{j k}$ denotes the number of hits (correct responses to left-sided stimuli), $n^{h}{ }_{j k}$ the total number of left-sided trials, and $\theta^{h}{ }_{j k}$ the hit rate attributable to participant $j$ in condition k. Similarly, $\mathrm{f}_{\mathrm{jk}}$ denotes the number of false alarms (incorrect responses to right-sided stimuli), $\mathrm{n}_{\mathrm{jk}}$ the total number of rightsided trials, and $\theta^{f}{ }_{\mathrm{jk}}$ the false alarm rate attributable to participant $\mathrm{j}$ in condition $\mathrm{k}$.

Hit and false alarm rates $\theta^{\text {h }}$ and $\theta^{f}$ were reparameterised in terms of sensitivity $\left(d^{\prime}\right)$ and response bias (c) in accordance with signal detection theory as shown below. ANOVA-like linear models [34] were 1124 placed on the d' and c estimates in order to estimate their variance components with respect to group 1125 membership and the experimental conditions. In contrast to the logistic model employed in Study 1, 1126 therefore, stimulus intensity was treated as a categorical rather than a continuous variable.

$$
\begin{gathered}
\theta_{j k}^{h}=\Phi\left(\left(\frac{\sqrt{2 d^{\prime}{ }_{j k}}}{2}\right)-c_{j k}\right) \\
\theta_{j k}^{f}=\Phi\left(-\left(\frac{\sqrt{2 d^{\prime}{ }_{j k}}}{2}\right)-c_{j k}\right) \\
d_{j k}^{\prime}=\alpha^{d^{\prime}} 0+\alpha^{d^{\prime}} R_{j}+\alpha^{d^{\prime}} F_{k}+\varepsilon_{j k}^{d^{\prime}} \\
c_{j k}=\alpha^{c} 0+\alpha^{c} R_{j}+\alpha^{c} F_{k}+\varepsilon_{j k}^{c}
\end{gathered}
$$

For both $d^{\prime}$ and $c$ :

$$
\begin{gathered}
\alpha 0 \sim N\left(\mu, \sigma^{0^{2}}\right) \\
\alpha R_{j} \sim N\left(0, \sigma^{R^{2}}\right) \\
\alpha F_{k} \sim N\left(0, \sigma_{f}^{F^{2}}\right)
\end{gathered}
$$




$$
\varepsilon_{j k} \sim N\left(0, \sigma^{\varepsilon^{2}}\right)
$$

For all $\sigma$ :

$$
\begin{aligned}
& \sigma \sim t(0,1, v), \sigma \geq 0 \\
& v \sim \operatorname{Gamma}(2,0.1)
\end{aligned}
$$

1129 where $\Phi$ is the CDF of the standard normal distribution, $\mathrm{R}$ represents a vector of random effects and

$1130 \mathrm{~F}$ is equivalent to a design matrix with $\mathrm{k}$ conditions grouped into $\mathrm{f}$ factors including a group factor (2

1131 levels), a modality factor (2 levels), and an intensity factor (4 levels). $\mu$ was estimated by averaging 1132 point estimates for the respective parameters over the whole dataset $\left(\mu^{d^{\prime}}=1.66, \mu^{c}=0.06\right)$.

1133

1134 RT distribution model (Study 2). Following [48], we employed a mixture model, comprising a uniform distribution to capture lapses and fast guesses, and a Weibull distribution to model RTs arising from attended trials, distributed as follows:

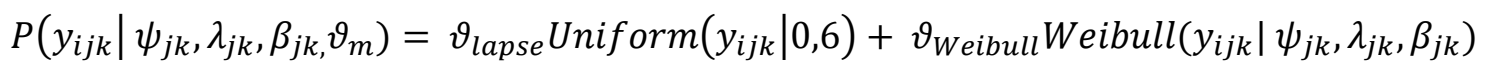

where again following [48] the Weibull scale parameter $\theta$ has been reparameterised as a rate parameter $\lambda$ for computational tractability using the transformation:

$$
\lambda_{j k}=\theta_{j k}^{-\beta_{j k}}
$$

and where $\mathrm{y}_{\mathrm{ijk}}$ represents the $\mathrm{i}^{\text {th }}$ response of the $\mathrm{j}^{\text {th }}$ participant in the $\mathrm{k}^{\text {th }}$ condition. The mixture weights associated with each model component were estimated from the data using the following

1141 uninformative priors:

$$
\begin{gathered}
\vartheta_{\text {lapse }} \sim \text { Uniform }(0,1) \\
\vartheta_{\text {Weibull }}=1-\vartheta_{\text {lapse }}
\end{gathered}
$$

1142 Linear models were placed upon the $\psi$ and $\lambda$ parameters of the Weibull distribution as follows:

$$
\begin{gathered}
\psi_{j k}=\alpha^{\psi} 0+\alpha^{\psi} R_{j}+\alpha^{\psi} F_{k}+\varepsilon_{j k}^{\psi} \\
\lambda_{j k}=\alpha^{\lambda} 0+\alpha^{\lambda} R_{j}+\alpha^{\lambda} F_{k}+\varepsilon_{j k}^{\lambda}
\end{gathered}
$$




$$
\beta_{j k} \sim N\left(2, \sigma^{\beta^{2}}\right)
$$

1143 for both $\psi$ and $\lambda$ :

$$
\begin{aligned}
& \alpha 0 \sim N\left(\mu^{0}, \sigma^{0^{2}}\right) \\
& \alpha R_{j} \sim N\left(0, \sigma^{R^{2}}\right) \\
& \alpha F_{k} \sim N\left(0, \sigma_{f}^{F^{2}}\right) \\
& \varepsilon_{j k} \sim N\left(0, \sigma^{\varepsilon^{2}}\right)
\end{aligned}
$$

1144 For all $\sigma$ :

$$
\begin{gathered}
\sigma \sim t(0,1, v), \sigma \geq 0 \\
v \sim \operatorname{Gamma}(2,0.1)
\end{gathered}
$$

1145 where $\mathrm{R}$ represents a vector of random effects and $\mathrm{F}$ is equivalent to a design matrix with $\mathrm{k}$ conditions 1146 grouped into f factors including a group factor (2 levels), a modality factor ( 2 levels), a laterality factor 1147 (2 levels) and an intensity factor (4 levels). $\mu^{0}$ was defined by averaging estimates for the respective 1148 parameters which had previously been derived using an unrestricted random effects model.

1150 As described in the Results section above, the model was rerun with the inclusion of the additional 1151 likelihood for "fast guesses" as follows:

$$
\begin{gathered}
P\left(y_{i j k} \mid \psi_{j k}, \lambda_{j k}, \beta_{j k}, \vartheta_{m}\right)=\vartheta_{\text {lapse }} \text { Uniform }\left(y_{i j k} \mid 0,6\right)+\vartheta_{\text {guess }} N\left(y_{i j k} \mid 0,1\right), y \\
\geq 0+\vartheta_{\text {Weibull }} \text { Weibull }\left(y_{i j k} \mid \psi_{j k}, \lambda_{j k}, \beta_{j k}\right) \\
\vartheta_{\text {lapse }} \sim \operatorname{Uniform}(0,1) \\
\vartheta_{\text {guess }} \sim \operatorname{Uniform}(0,1) \\
\vartheta_{\text {Weibull }}=1-\left(\vartheta_{\text {lapse }}+\vartheta_{\text {guess }}\right)
\end{gathered}
$$

\section{Study 3 agents}

Agents designed to simulate datasets on the basis of the Study 1 and Study 2 tasks were developed using Matlab (R2018a, The Mathworks). 
Simulated Study 1 agent. The agent in the Study 1 task used a simple Bayesian model to detect and respond to stimuli occurring as part of an adaptive staircase. In all respects other than the symbolic nature of stimulus and response the task was identical to that carried out by human participants in Study 1.

Stimulus. The stimulus was represented by an input describing its intensity in decibels, which was then translated into a location $\mu^{\text {signal }}$ on a sensitivity / detectability scale from 0 (undetectable) to 3 (clearly detectable). The translation was achieved by means of a sigmoid curve fitted to the relationship between stimulus intensity and $d^{\prime}$ in the observed data from the control group in Study 1: the translation therefore differed for the two modalities. As in the real task, the stimulus occurred at random in one of two possible intervals. A 'noise' event, occurring in the other interval, was represented on the sensitivity scale by the location $\mu^{\text {noise }}=0$.

Generative model. The agent initially made a prior prediction about the likely detectability $(\theta)$ of the impending signal, informed by knowledge about the operation of the staircase and about stimulus detectability during previous trials. This prior was distributed as:

$$
P(\theta) \sim N\left(\mu^{\text {prior }}, \sigma^{\text {prior }^{2}}\right)
$$

1172 where the initial value of $\mu^{\text {prior }}=3$, and subsequent values were based on the posterior probabilities 1173 of previous trials. The precision of the prior was given a default value of 1.

The agent then received noisy inputs associated with the respective intervals as follows:

$$
\begin{gathered}
\text { data }^{\text {signal }} \sim N\left(\mu^{\text {signal }}, \sigma^{\text {signal }^{2}}\right) \\
\text { data }^{\text {noise }} \sim N\left(\mu^{\text {noise }}, \sigma^{\text {noise }}{ }^{2}\right)
\end{gathered}
$$


1176 A likelihood function was calculated for each interval, giving the probability of the data acquired during

1177 that interval given the possible range of signal detectability as follows:

$$
P(\text { data } \mid \theta, \text { interval })=N\left(\text { data }, \theta, \sigma^{\text {data }}{ }^{2}\right)
$$

1178

For simplicity, $\sigma^{\text {signal }}=\sigma^{\text {noise }}=\sigma^{\text {data }}$ in this application and represents a reparameterisation of a default precision of 4 for all likelihood functions.

Recognition model. The generative model was then inverted according to Bayes Theorem to give the posterior probability of signal detectability, given the data, for each interval:

$$
P(\theta \mid \text { data }, \text { interval })=\frac{P(\text { data } \mid \theta, \text { interval }) P(\theta)}{P(\text { data } \mid \text { interval })}
$$

where the overall probability of the data for each interval is given by:

$$
P(\text { data } \mid \text { interval })=\int P(\text { data } \mid \theta, \text { interval }) P(\theta) d \theta
$$

The posterior distributions for each interval were treated as percepts upon which the agent based its responses. Figure 10 (top panels) shows the prior, likelihood and posterior distributions for both intervals (signal occurring in Interval 1 , data shown in each interval as a black vertical line) in a hypothetical trial occurring at the outset of a staircase where the expected detectability of the signal is at its maximum of 3.

A posterior distribution placing little or no probability $(P(\theta=0)<0.01)$ at 0 on the detectability scale was considered to be unambiguously detectable. A posterior distribution placing $50 \%$ or more probability below 0.25 on the detectability scale was considered to be undetectable. This cut-off was relatively arbitrary, but was chosen because it produced similar behaviour in the agent to that observed in the human participants. 
1196

1197

1198

1199

1200

1201

1202

1203

1204 data:

$$
P(\text { interval } \mid \text { data })=\frac{P(\text { interval }) P(\text { data } \mid \text { interval })}{\sum P(\text { interval }) P(\text { data } \mid \text { interval })}
$$

1205 process.

$$
P(\text { interval })=0.5
$$

to give the normalised posterior probability that the signal had occurred in each interval given the The agent then drew its response from a Bernoulli distribution with a rate reflecting the relative posterior probabilities.

Finally, the prior expectation of stimulus detectability $P(\theta) \sim N\left(\mu^{\text {prior }}, \sigma^{\text {prior2 }}\right)$ was updated based on the outcome of the trial. This was achieved by updating $\mu^{\text {prior }}$ with the maximum a posteriori estimate of the posterior distribution. The precision of the prior remained constant at 1 throughout the task for the sake of simplicity. Figure 10 (lower panel) shows a staircase generated by the agent using this 

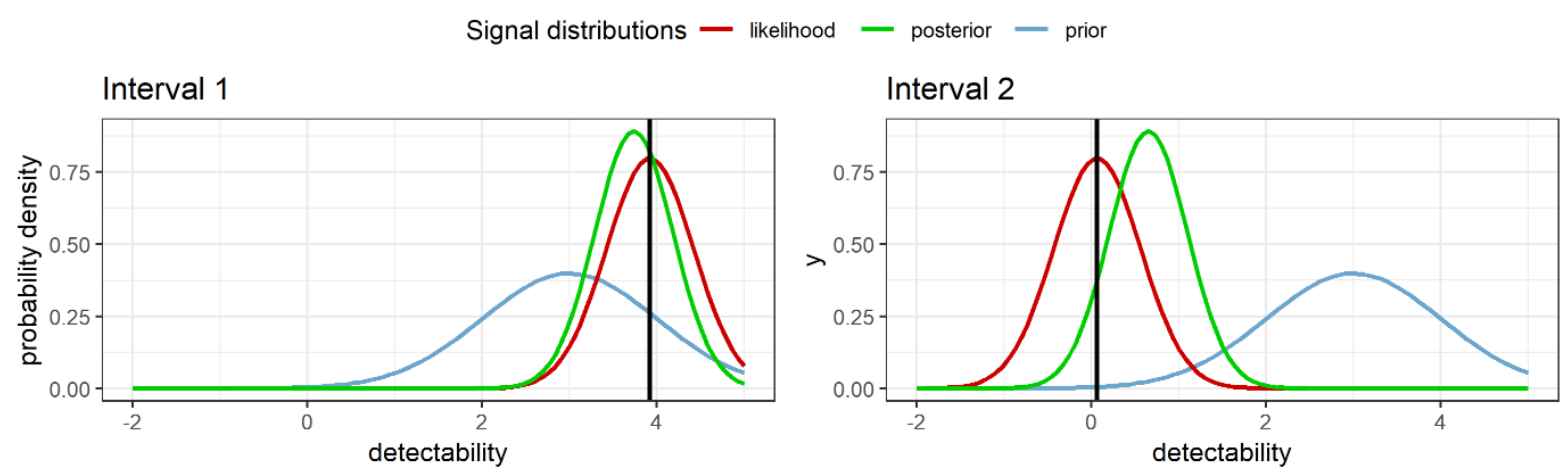

Adaptive staircase examples (left somatic control data)

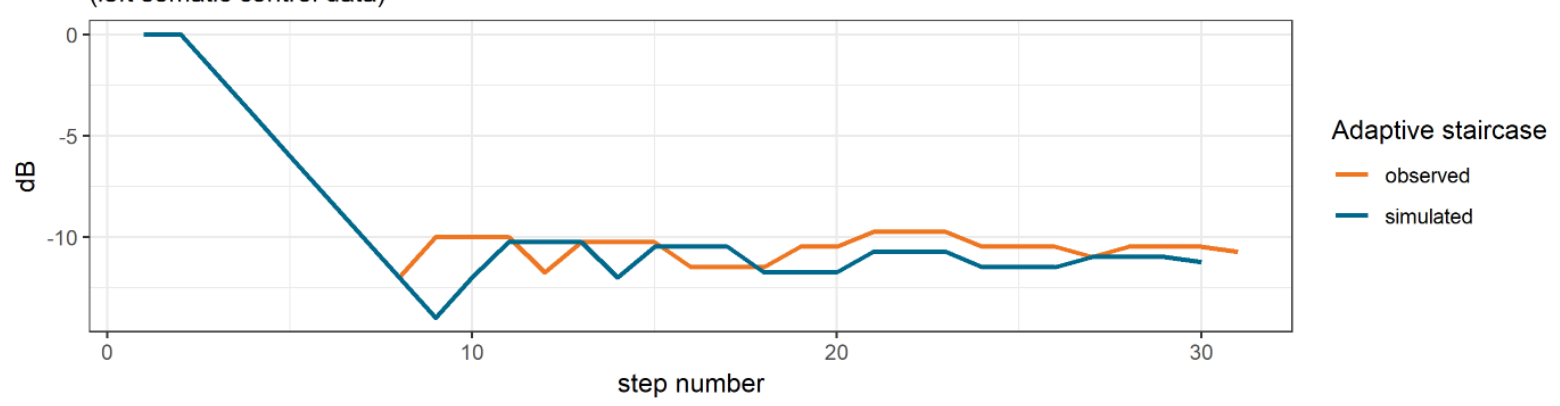

Figure 10. A simulated trial and resulting staircase arising as part of the simulation of Study 1. Upper

panels show the prior, likelihood and posterior distributions for the two intervals in a hypothetical

trial occurring at the outset of a staircase where the expected detectability of the signal is at its maximum of 3 . The signal occurs during Interval 1, and for each interval the (noisy) input is shown as a black, vertical line. As can be seen, the posterior distribution ("percept") arising from the Interval 1 input is detectable, placing effectively no probability at zero. The corresponding "percept" arising from the Interval 2 input is ambiguous, with substantial probability at or below zero. On the basis of these outcomes, the agent would select "interval 1" with a probability of 1 . The lower panel shows a staircase simulated using this process (randomly selected from the total 128 staircases generated in the process of simulating a Study 1 dataset) plotted against a staircase (matched by group and condition) randomly selected from the observed Study 1 data.

Simulated Study 2 agent. The Study 2 task differed from that in Study 1 in employing two distinct staircases, one modulating the intensity of stimuli occurring on the left, and one modulating the 
intensity of stimuli occurring on the right. Either staircase could be in operation during any given trial, and the task of the participant was to make a judgement regarding which side of space a stimulus occurred in that trial, given current expectations about the likely intensity of a left-sided stimulus versus the likely intensity of a right-sided stimulus versus noise. In order to model this task, it was therefore necessary for the agent to develop two independent models, one relating to each staircase.

Stimulus. The representation of the stimulus was similar to that in the Study 1 simulation, and similarly employed modality-specific sigmoid transformations based upon the observed control sensitivity data to transform a representation of stimulus intensity (dB) into a location $\mu^{\text {signal }}$ on a sensitivity / detectability scale. The detectability scale ran from -3 (highly detectable left-sided stimulus) to 3 (highly detectable right-sided stimulus) with the range near 0 representing low detectability / high uncertainty. Staircase laterality in any given trial was chosen at random, and a "noise" event occurring in the alternative laterality was represented on the detectability scale by the location $\mu^{\text {noise }}=0$.

Generative model. The agent in this task relied upon a more complex set of prior expectations resulting from the additional information provided by the existence of two independently varying staircases. The priors for the signal differed depending on staircase laterality, and additionally a prior was created to represent the expectation of noise:

$$
\begin{gathered}
P(\theta \mid \text { laterality, input }=\text { signal }) \sim N\left(\mu_{\text {laterality }}^{\text {signal }}, \sigma^{\text {prior }^{2}}\right) \\
P(\theta \mid \text { input }=\text { noise }) \sim N\left(\mu^{\text {noise }}, \sigma^{\text {prior }^{2}}\right)
\end{gathered}
$$

The initial values of $\mu^{\text {signal }}$ were -3 and 3 depending on laterality, and subsequent values were based on the posterior probabilities of previous trials. $\mu^{\text {noise }}$ remained constant at 0 throughout the task. As before the precision of all priors had a default value of 1 . 
As before, the agent received noisy inputs associated respectively with each side of space as follows:

$$
\begin{gathered}
\text { data }^{\text {signal }} \sim N\left(\mu^{\text {signal }}, \sigma^{\text {signal }^{2}}\right) \\
\text { data }^{\text {noise }} \sim N\left(\mu^{\text {noise }}, \sigma^{\text {noise }}{ }^{2}\right)
\end{gathered}
$$

A likelihood function was calculated for each laterality and each possible input (signal or noise), as follows:

$$
P(\text { data } \mid \theta, \text { laterality, input })=N\left(\text { data }, \theta, \sigma^{\text {data }}{ }^{2}\right)
$$

1255

1256

1257

1258

1259

As before, $\sigma^{\text {signal }}=\sigma^{\text {noise }}=\sigma^{\text {data }}$ in this application and represents a reparameterisation of a default precision of 4 for all likelihood functions.

Recognition model. As before, the generative model for the signal distributions was inverted according to Bayes Theorem to calculate the posterior probability of signal detectability, given the data, for each interval. Note that as in the previous model, this procedure assumes that the data arise from signal rather than from noise:

$$
\begin{aligned}
& P(\theta \mid \text { data, laterality, input }=\text { signal }) \\
& \qquad=\frac{P(\text { data } \mid \theta, \text { laterality, input }=\text { signal }) P(\theta \mid \text { laterality, } \text { input }=\text { signal })}{P(\text { data } \mid \text { laterality, } \text { input }=\text { signal })}
\end{aligned}
$$

where the probability of the data given laterality is given by:

$$
\begin{gathered}
P(\text { data } \mid \text { laterality, input }=\text { signal })=\int P(\text { data } \mid \theta, \text { laterality, } \text { input } \\
=\text { signal }) P(\theta \mid \text { laterality, input }=\text { signal }) d \theta
\end{gathered}
$$

As before, the posterior distributions of signal detectability for each laterality were treated as percepts upon which the agent based its responses. The same criteria for detectability and undetectability were employed as in the Study 1 simulations.

Response decisions. When a percept was uniquely detectable on one side of space, the agent gave the response associated with that laterality with a probability of 1 . When both percepts were undetectable, the agent "guessed" at random, drawing its response from a Bernoulli distribution with 
a rate of 0.5 . In the case of ambiguity, the agent combined the signal and noise likelihoods calculated in the previous section with the known prior probability of a signal occurring on either side of space:

$$
P(\text { input }=\text { signal } \mid \text { laterality })=0.5
$$
the data, independently for each laterality. For each laterality:

$$
\begin{aligned}
& P(\text { input }=\text { signal } \mid \text { data }, \text { laterality }) \\
& \qquad=\frac{P(\text { data } \mid \text { input }=\text { signal, laterality }) P(\text { input }=\text { signal } \mid \text { laterality })}{\sum_{i=1}^{\text {input }} P(\text { data } \mid \text { input }, \text { laterality })}
\end{aligned}
$$

Importantly, this process yields two distinct posterior estimates, one based on evidence from the left side of space, and one based on evidence from the right. This innovation allows the agent to rely preferentially on evidence from one side of space or the other, although in the current (default) model final estimates of the averaged posterior probability

$$
\begin{aligned}
& P(\text { input }=\text { signal } \mid \text { data }, \text { laterality }=\text { left }) \\
& \quad=1-P(\text { input }=\text { signal } \mid \text { data }, \text { laterality }=\text { right })
\end{aligned}
$$

were calculated using a model-averaging procedure with weights of 0.5 for each model. The agent then drew its response from a Bernoulli distribution with a rate reflecting the relative posterior probabilities.

Finally, the prior expectation of stimulus detectability

$$
P(\theta \mid \text { laterality, input }=\text { signal }) \sim N\left(\mu_{\text {laterality }}^{\text {signal }}, \sigma^{\text {prior }^{2}}\right)
$$

was updated based on the outcome of the trial. This was achieved by updating $\mu^{\text {signal }}$ with the maximum a posteriori estimate of the posterior distribution for the laterality selected by the agent as the correct one for that trial. As before, the precision of all priors remained constant at 1 throughout the task.

The agents were then modified in order to address the questions posed at the outset of Study 3. 
Lapse variants. A simple model of attentional lapses was incorporated into the Study 1 agent described above. A proportion of trials occurring with rate $\lambda$ were designated "lapse trials". A lapse trial was distinguished from fully attended trials in that the parameters, $\sigma^{\text {signal }}=\sigma^{\text {noise }}=\sigma^{\text {data }}=10$ (i.e., the precision of the likelihoods was 0.01 rather than 4 as in fully attended trials). The practical effect of this change in precision is that signal and noise likelihoods become indistinguishable from each other even given a highly detectable input, and that due to the imprecision of the data the posterior distributions are to all intents and purposes indistinguishable from their priors. The attentional status of the agent in any given trial was determined by a draw from a Bernoulli distribution with rate $\lambda$.

The data presented in the Results section are derived from a simulation in which $\lambda^{\text {left }}=0.06$ and $\lambda^{\text {right }}$ $=0.01$ for 16 "depressed" simulated participants, and $\lambda^{\text {left }}=0.01$ and $\lambda^{\text {right }}=0.03$ for 16 "healthy controls".

A similar lapse model, with both "depressed" and "control" $\lambda$ values identical to the Study 1 agent, was incorporated into the Study 2 agent. Given that, in contrast to the Study 1 simulation, the agent "attended" to both sides of space simultaneously, the precision of the likelihoods was reduced for one side of space only during a lapse trial, unless a lapse coincidentally occurred on both sides at once.

The Study 2 task also differed from the previous task in that its interleaved staircases afforded redundant information about each laterality based on evidence from the other, and therefore compensatory strategies could in principle be employed in response to lateralised attentional deficits. On this basis three variants of the lapsing agent were developed. 
Intuited lapses variant (Figure 7, Panel D). The agent was able to accurately "intuit" lapses based on the precision of the likelihood. During trials in which a lapse occurred, the agent adjusted its prior expectations about signal laterality accordingly:

$$
P(\text { laterality }=\text { left } \mid \text { lapse })=\frac{\lambda^{\text {left }}}{\lambda^{\text {left }}+\lambda^{\text {right }}}
$$

Compensated lapses variant (Figure 7, Panel E)._The agent could not accurately intuit lapses, or otherwise distinguish between lapses and trials in which there was no detectable signal. The agent therefore employed the adjusted laterality prior described in the previous model as a biased guessing rule in any trial yielding no reliable data (those in which the likelihood was too vague to affect the prior, and those in which percepts on both sides of space were undetectable). Note that this strategy is overcompensatory in its overgeneral use of the guessing rule.

For each variant of the lapsing Agent 2 described above, a dataset was derived from a simulation in which $\lambda^{\text {left }}=0.06$ and $\lambda^{\text {right }}=0.01$ for 12 "depressed" simulated participants, and $\lambda^{\text {left }}=0.01$ and $\lambda^{\text {right }}=$ 0.03 for 12 "healthy controls".

Resource conservation variants. The original Study 2 agent was modified to reduce the precision of the likelihood and prior on the left side of space commensurately with the expectation of the agent that that the left-sided percept would be difficult to detect. This strategy was implemented by generating a prediction of the distribution of the next left-sided signal percept given the current prior and predicted data. The reduction in precision on the left (parameterised as $\Delta \sigma^{\text {unattended }}$ ) was a function of the probability density of the predicted posterior at 0 on the detectability scale as follows:

$$
\Delta \sigma^{\text {unattended }}=\frac{1}{2} P(\theta=0 \mid \text { predicted data })^{1.5}
$$

In order to maintain the relative precisions of prior and likelihood, they were adjusted using the following formula (reparameterised as a standard deviation) 


$$
\sigma^{\text {unattended }}=\sigma^{\text {attended }}+\left(\Delta \sigma^{\text {unattended }} \sigma^{\text {attended }}\right)
$$

As a result, when a detectable left-sided percept was expected the precisions did not differ across space, but as expected left-sided stimulus detectability reduced, precision on the left side reduced proportionally. At the point where the left-sided stimulus was expected to be completely undetectable, left-sided precision was about $1 / 2$ of the precision on the right. Three versions of this model were simulated as follows:

Uncompensated resource conservation variant (Figure 7, Panel F)._No compensatory strategies were employed.

Precision-weighted resource conservation variant (Figure 7, Panel G). The agent used precision-weighting to rely increasingly on evidence generated from the right side of space as precision to the left was reduced, using the formula

$$
w=\frac{\tau^{\text {right }}}{\left(\tau^{\text {left }}+\tau^{\text {right }}\right)}
$$

where $\tau$ is the precision of the posterior distributions and $w$ is the weighting used for the rightlateralised posterior probability $\mathrm{P}$ (input=signal|data) relative to the left. In addition, in trials in which neither stimulus was unambiguously detectable, but the stimulus on the right was unambiguously undetectable, the agent adjusted the guess rate to $1-\mathrm{w}$ (i.e., when no detectable information is available on the attended right side of space, the agent increases its belief that the signal occurred on the left).

Overcompensated resource-conservation variant (Figure 7, Panel H). This model was identical to the previous one except that $w$ was multiplied by 1.2 to provide an over-compensatory precisionweighting strategy. 
1359

1360

1361

1362

1363

1364

1365

1366

1367

1368

1369

1370

1371

1372

1373

1374

1375

Constant deficit variants. Both the Study 1 and Study 2 agents were modified by the addition of a small, constant amount of noise to the left-sided likelihoods and priors. A number of experiments were undertaken with respect to the magnitude and consistency of the increase in noise, but these did not influence the patterns seen in the resulting data, only the magnitude of the resulting effects.

\section{False percept variants}

Unmodulated false percept variant (Figure 7, Panel I). The precision of the left-sided expectation of a signal was constant.

Modulated false percept variant (Figure 7, Panel J). The precision of the left-sided expectation of a signal increased commensurately with the expectation that the right-sided stimulus would be undetectable as follows (where the probability refers to signal detectability on the right side of space):

$$
\sigma^{\text {left signal prior }}=1-P(\theta=0 \mid \text { predicted data })
$$

\section{Acknowledgements}

We would like to acknowledge the support of our group and the invaluable contribution of all our participants.

\section{References}

1. Bylsma LM, Morris BH, Rottenberg J. A meta-analysis of emotional reactivity in major depressive disorder. Clinical psychology review. 2008;28(4):676-91.

2. Rottenberg J, Joormann J, Brozovich F, Gotlib IH. Emotional intensity of idiographic sad memories in depression predicts symptom levels 1 year later. Emotion. 2005;5(2):238. 
1381

1382

1383

1384

1385

1386

1387

1388

1389

1390

1391

1392

1393

1394

1395

1396

1397

1398

1399

1400

1401

1402

1403

1404

3. Mula M, Pini S, Cassano GB. The neurobiology and clinical significance of depersonalization in mood and anxiety disorders: A critical reappraisal. Journal of affective disorders. 2007;99(1-3):91-9.

4. Fitzgerald PJ. Gray colored glasses: is major depression partially a sensory perceptual disorder? Journal of affective disorders. 2013;151(2):418-22.

5. Bubl E, Ebert D, Kern E, van Elst LT, Bach M. Effect of antidepressive therapy on retinal contrast processing in depressive disorder. The British Journal of Psychiatry. 2012;201(2):151-8.

6. Bubl E, Kern E, Ebert D, Bach M, Van Elst LT. Seeing gray when feeling blue? Depression can be measured in the eye of the diseased. Biological psychiatry. 2010;68(2):205-8.

7. Bubl E, Kern E, Ebert D, Riedel A, van Elst LT, Bach M. Retinal dysfunction of contrast processing in major depression also apparent in cortical activity. European archives of psychiatry and clinical neuroscience. 2015;265(4):343-50.

8. Bubl E, Tebartz Van Elst L, Gondan M, Ebert D, Greenlee MW. Vision in depressive disorder. The World Journal of Biological Psychiatry. 2009;10(4-2):377-84.

9. Malone J, Hemsley D. Lowered responsiveness and auditory signal detectability during depression. Psychological Medicine. 1977;7(4):717-22.

10. Yovell Y, Sackeim HA, Epstein DG, Prudic J, Devanand D, Settembrino JM, et al. Hearing Loss and Asymmetry in Major. Neurosciences. 1995;7:82-9.

11. Schwenzer M, Zattarin E, Grözinger M, Mathiak K. Impaired pitch identification as a potential marker for depression. BMC psychiatry. 2012;12(1):32.

12. Croy I, Symmank A, Schellong J, Hummel C, Gerber J, Joraschky P, et al. Olfaction as a marker for depression in humans. Journal of affective disorders. 2014;160:80-6.

13. Khil L, Rahe C, Wellmann J, Baune BT, Wersching H, Berger K. Association between major depressive disorder and odor identification impairment. Journal of affective disorders. 2016;203:332-

8. 
14. Pause BM, Miranda A, Göder R, Aldenhoff JB, Ferstl R. Reduced olfactory performance in patients with major depression. Journal of psychiatric research. 2001;35(5):271-7.

15. Steiner JE, Lidar-Lifschitz D, Perl E. Taste and odor: reactivity in depressive disorders, a multidisciplinary approach. Perceptual and motor skills. 1993;77(3_suppl):1331-46.

16. Dichter GS, Smoski MJ, Kampov-Polevoy AB, Gallop R, Garbutt JC. Unipolar depression does not moderate responses to the Sweet Taste Test. Depression and anxiety. 2010;27(9):859-63.

17. Swiecicki L, Zatorski P, Bzinkowska D, Sienkiewicz-Jarosz H, Szyndler J, Scinska A. Gustatory and olfactory function in patients with unipolar and bipolar depression. Progress in NeuroPsychopharmacology and Biological Psychiatry. 2009;33(5):827-34.

18. Schachter S, Singer JE. Cognitive, social, and physiological determinants of emotional state. Psychological review. 1962;69:379-99.

19. Strigo IA, Craig AD. Interoception, homeostatic emotions and sympathovagal balance. Philosophical Transactions of the Royal Society B: Biological Sciences. 2016;371(1708):20160010.

20. Critchley HD, Garfinkel SN. Interoception and emotion. Current opinion in psychology. 2017;17:7-14

21. Adler G, Gattaz WF. Pain perception threshold in major depression. Biological psychiatry. 1993;34(10):687-9.

22. Marazziti D, Castrogiovanni P, Rossi A, Rosa C, Ghione S, Di Muro A, et al. Pain threshold is reduced in depression. International Journal of Neuropsychopharmacology. 1998;1(1):45-8.

23. Bär K-J, Terhaar J, Boettger MK, Boettger S, Berger S, Weiss T. Pseudohypoalgesia on the skin: a novel view on the paradox of pain perception in depression. Journal of clinical psychopharmacology. 2011;31(1):103-7.

24. Ben-Tovim DI, Schwartz MS. Hypoalgesia in depressive illness. The British Journal of Psychiatry. 1981;138(1):37-9. 
25. Klauenberg S, Maier C, Assion H-J, Hoffmann A, Krumova EK, Magerl W, et al. Depression and changed pain perception: hints for a central disinhibition mechanism. Pain. 2008;140(2):332-43.

26. Marsala SZ, Pistacchi M, Tocco P, Gioulis M, Fabris F, Brigo F, et al. Pain perception in major depressive disorder: A neurophysiological case-control study. Journal of the neurological sciences. 2015;357(1-2):19-21.

27. Terhaar J, Boettger MK, Schwier C, Wagner G, Israel A-K, Bär K-J. Increased sensitivity to heat pain after sad mood induction in female patients with major depression. European Journal of Pain. 2010;14(5):559-63.

28. Moroz B, Nuller I, Ustimova I, Andreev B. Study of pain sensitivity based on the indicators of electro-odontometry in patients with depersonalization and depressive disorders. Zhurnal nevropatologii i psikhiatrii imeni SS Korsakova (Moscow, Russia: 1952). 1990;90(10):81-2.

29. Mialet J-P, Pope H, Yurgelun-Todd D. Impaired attention in depressive states: a non-specific deficit? Psychological Medicine. 1996;26(5):1009-20.

30. Farrin L, Hull L, Unwin C, Wykes T, David A. Effects of depressed mood on objective and subjective measures of attention. The Journal of neuropsychiatry and clinical neurosciences. 2003;15(1):98-104.

31. Koster EH, De Raedt R, Goeleven E, Franck E, Crombez G. Mood-congruent attentional bias in dysphoria: maintained attention to and impaired disengagement from negative information. Emotion. 2005;5(4):446

32. Gelman A, Carlin JB, Stern HS, Dunson DB, Vehtari A, Rubin DB. Bayesian data analysis: Chapman and Hall/CRC; 2013.

33. Lee MD, Wagenmakers E-J. Bayesian cognitive modeling: A practical course: Cambridge university press; 2014.

34. Kruschke J. Doing Bayesian data analysis: A tutorial with R, JAGS, and Stan: Academic Press; 2014. 
1454 35. Levitt H. Transformed up-down methods in psychoacoustics. The Journal of the Acoustical

1455

1456

1457

1458

1459

1460

1461

1462

1463

1464

1465

1466

1467

1468

1469

1470

1471

1472 Society of America. 1971;49(2):Suppl 2:467+.

36. Leek MR. Adaptive procedures in psychophysical research. Perception \& psychophysics. 2001;63(8):1279-92.

37. Dellaportas P, Forster JJ, Ntzoufras I. On Bayesian model and variable selection using MCMC. Statistics and Computing. 2002;12(1):27-36.

38. Prins N. The psychometric function: The lapse rate revisited. Journal of Vision. 2012;12(6):25-

39. Wichmann FA, Hill NJ. The psychometric function: I. Fitting, sampling, and goodness of fit. Perception \& psychophysics. 2001;63(8):1293-313.

40. García-Pérez MA, Alcalá-Quintana R. Interval bias in 2AFC detection tasks: sorting out the artifacts. Attention, Perception, \& Psychophysics. 2011;73(7):2332-52.

41. Yang X, Ma X, Huang B, Sun G, Zhao L, Lin D, et al. Gray matter volume abnormalities were associated with sustained attention in unmedicated major depression. Comprehensive psychiatry. 2015;63:71-9.

42. Van Der Meere J, Börger N, Van Os T. Sustained attention in major unipolar depression. Perceptual and motor skills. 2007;104(3_suppl):1350-4.

43. Kelly AM, Uddin LQ, Biswal BB, Castellanos FX, Milham MP. Competition between functional brain networks mediates behavioral variability. Neurolmage. 2008;39(1):527-37.

44. Bellgrove MA, Hester R, Garavan H. The functional neuroanatomical correlates of response variability: evidence from a response inhibition task. Neuropsychologia. 2004;42(14):1910-6.

45. Weissman DH, Roberts KC, Visscher KM, Woldorff MG. The neural bases of momentary lapses in attention. Nature neuroscience. 2006;9(7):971-8.

46. Green DM, Swets JA. Signal detection theory and psychophysics: Wiley New York; 1966.

47. Jeffreys $\mathrm{H}$. The theory of probability: OUP Oxford; 1961. 
48. Rouder JN, Lu J, Speckman P, Sun D, Jiang Y. A hierarchical model for estimating response time distributions. Psychonomic Bulletin \& Review. 2005;12(2):195-223.

49. García-Pérez MA, Alcalá-Quintana R. The difference model with guessing explains interval bias in two-alternative forced-choice detection procedures. Journal of sensory studies. 2010;25(6):876-98. 50. García-Pérez MA, Alcalá-Quintana R. The indecision model of psychophysical performance in dual-presentation tasks: Parameter estimation and comparative analysis of response formats. Frontiers in psychology. 2017;8:1142.

51. Friston K. The free-energy principle: a unified brain theory? Nature reviews neuroscience. 2010;11(2):127.

52. Clark A. Whatever next? Predictive brains, situated agents, and the future of cognitive science. Behavioral and brain sciences. 2013;36(3):181-204.

53. Knill DC, Pouget A. The Bayesian brain: the role of uncertainty in neural coding and computation. Trends in neurosciences. 2004;27(12):712-9.

54. Vossel S, Mathys C, Daunizeau J, Bauer M, Driver J, Friston KJ, et al. Spatial attention, precision, and Bayesian inference: a study of saccadic response speed. Cerebral cortex. 2013;24(6):1436-50.

55. Feldman H, Friston K. Attention, uncertainty, and free-energy. Frontiers in human neuroscience. 2010;4:215.

56. Pizzagalli DA, Jahn AL, O'Shea JP. Toward an objective characterization of an anhedonic phenotype: a signal-detection approach. Biological psychiatry. 2005;57(4):319-27.

57. Brooks JL, Darling S, Malvaso C, Della Sala S. Adult developmental trajectories of pseudoneglect in the tactile, visual and auditory modalities and the influence of starting position and stimulus length. Brain and cognition. 2016;103:12-22.

58. Bowers D, Heilman KM. Pseudoneglect: Effects of hemispace on a tactile line bisection task. Neuropsychologia. 1980;18(4-5):491-8. 
59. Benwell CS, Harvey M, Thut G. On the neural origin of pseudoneglect: EEG-correlates of shifts in line bisection performance with manipulation of line length. Neurolmage. 2014;86:370-80.

60. De Schotten MT, Dell'Acqua F, Forkel SJ, Simmons A, Vergani F, Murphy DG, et al. A lateralized brain network for visuospatial attention. Nature neuroscience. 2011;14(10):1245.

61. Liotti M, Mayberg HS. The role of functional neuroimaging in the neuropsychology of depression. Journal of Clinical and Experimental Neuropsychology. 2001;23(1):121-36.

62. Liotti M, Sava D, Rizzolatti G, Caffarra P. Differential hemispheric asymmetries in depression and anxiety: a reaction-time study. Biological psychiatry. 1991;29(9):887-99.

63. Bruder GE, Quitkin FM, Stewart JW, Martin C, VogImaier MM, Harrison WM. Cerebral laterality and depression: differences in perceptual asymmetry among diagnostic subtypes. Journal of abnormal psychology. 1989;98(2):177.

64. Bartolomeo $\mathrm{P}$, Thiebaut De Schotten $\mathrm{M}$, Chica AB. Brain networks of visuospatial attention and their disruption in visual neglect. Frontiers in human neuroscience. 2012;6:110.

65. He BJ, Snyder AZ, Vincent JL, Epstein A, Shulman GL, Corbetta M. Breakdown of functional connectivity in frontoparietal networks underlies behavioral deficits in spatial neglect. Neuron. 2007;53(6):905-18.

66. Corbetta M, Shulman GL. Control of goal-directed and stimulus-driven attention in the brain. Nature reviews Neuroscience. 2002;3(3):201-15.

67. Downar J, Crawley AP, Mikulis DJ, Davis KD. The effect of task relevance on the cortical response to changes in visual and auditory stimuli: an event-related fMRI study. Neurolmage. 2001;14(6):1256-67.

68. Corbetta M, Patel G, Shulman GL. The reorienting system of the human brain: from environment to theory of mind. Neuron. 2008;58(3):306-24.

69. Doll RJ, Veltink PH, Buitenweg JR. Observation of time-dependent psychophysical functions and accounting for threshold drifts. Attention, Perception, \& Psychophysics. 2015;77(4):1440-7. 
70. Leek MR, Hanna TE, Marshall L. An interleaved tracking procedure to monitor unstable psychometric functions. The Journal of the Acoustical Society of America. 1991;90(3):1385-97. 71. Segal ZV, Teasdale J. Mindfulness-based cognitive therapy for depression: Guilford Publications; 2018.

72. Kenny M, Williams J. Treatment-resistant depressed patients show a good response to mindfulness-based cognitive therapy. Behaviour research and therapy. 2007;45(3):617-25.

73. Kuyken W, Hayes R, Barrett B, Byng R, Dalgleish T, Kessler D, et al. Effectiveness and costeffectiveness of mindfulness-based cognitive therapy compared with maintenance antidepressant treatment in the prevention of depressive relapse or recurrence (PREVENT): a randomised controlled trial. The Lancet. 2015;386(9988):63-73.

74. First MB, Spitzer RL, Gibbon M, Williams JB. Structured clinical interview for DSM-IV-TR axis I disorders, research version, patient edition. SCID-I/P New York, NY; 2002.

75. Beck AT, Steer RA, Ball R, Ranieri W. Comparison of Beck Depression Inventories -IA and -II in psychiatric outpatients. J Pers Assess. 1996;67(3):588-97.

76. Beck AT, Epstein N, Brown G, Steer RA. An inventory for measuring clinical anxiety: psychometric properties. Journal of consulting and clinical psychology. 1988;56(6):893-7.

77. Beck AT, Steer RA, Brown GK. Manual for the Beck Depression Inventory-Second Edition. San Antonio, TX: The Psychological Corporation. 1996.

78. Beck AT, Steer R. Beck Anxiety Inventory Manual. The Psychological Corporation. San Antonio, TX. 1993.

79. Brainard DH. The Psychophysics Toolbox. Spatial vision. 1997;10(4):433-6.

80. Pelli DG. The VideoToolbox software for visual psychophysics: transforming numbers into movies. Spatial vision. 1997;10(4):437-42.

81. Warriner AB, Kuperman V, Brysbaert M. Norms of valence, arousal, and dominance for 13,915 English lemmas. Behavior research methods. 2013;45(4):1191-207. 
1553 82. Plummer M. JAGS version 4.3. 0 user manual. 28 June 2017. 2017.

1554 83. Team RC. R: A language and environment for statistical computing [Internet]. Vienna, Austria; 1555 2014. 2017.

1556 84. Denwood MJ. runjags: An R package providing interface utilities, model templates, parallel 1557 computing methods and additional distributions for MCMC models in JAGS. Journal of Statistical 1558 Software. 2016;71(9):1-25.

1559 85. Plummer M, Best N, Cowles K, Vines K. CODA: convergence diagnosis and output analysis for 1560 MCMC. R News 6 (1), 7-11 (2006). 2006

1561 86. Gelman A, Rubin DB. Inference from iterative simulation using multiple sequences. Statistical 1562 science. 1992;7(4):457-72.

1563 87. Wickham H. ggplot2: elegant graphics for data analysis: Springer; 2016.

1564 88. Kassambara A. ggpubr: "ggplot2" based publication ready plots. R package version 01. 2017;6. 1565 89. Carlin BP, Chib S. Bayesian model choice via Markov chain Monte Carlo methods. Journal of 1566 the Royal Statistical Society: Series B (Methodological). 1995;57(3):473-84. 


\section{Supporting information}

\section{Section 1: Medication-free analyses}

1570 Study 1 GLMM with logistic link function. The analyses described in Study 1 were repeated including $n=10$ unmedicated depressed participants and $n=16$ healthy controls. Outcomes did not differ from the whole-group analyses described in the main study. Figure S1 shows the model outcomes. Table S1 shows the outcomes of the variable selection procedure, which do not differ from those derived from the whole-group variable selection analysis. As in the main analysis, when the lapse rate was restricted to zero disproportionate left-sided lapses among the depressed sample contributed to a credible interaction between group $\mathrm{x}$ laterality in the slope parameter which was abolished by the inclusion of a lapse parameter.

1578
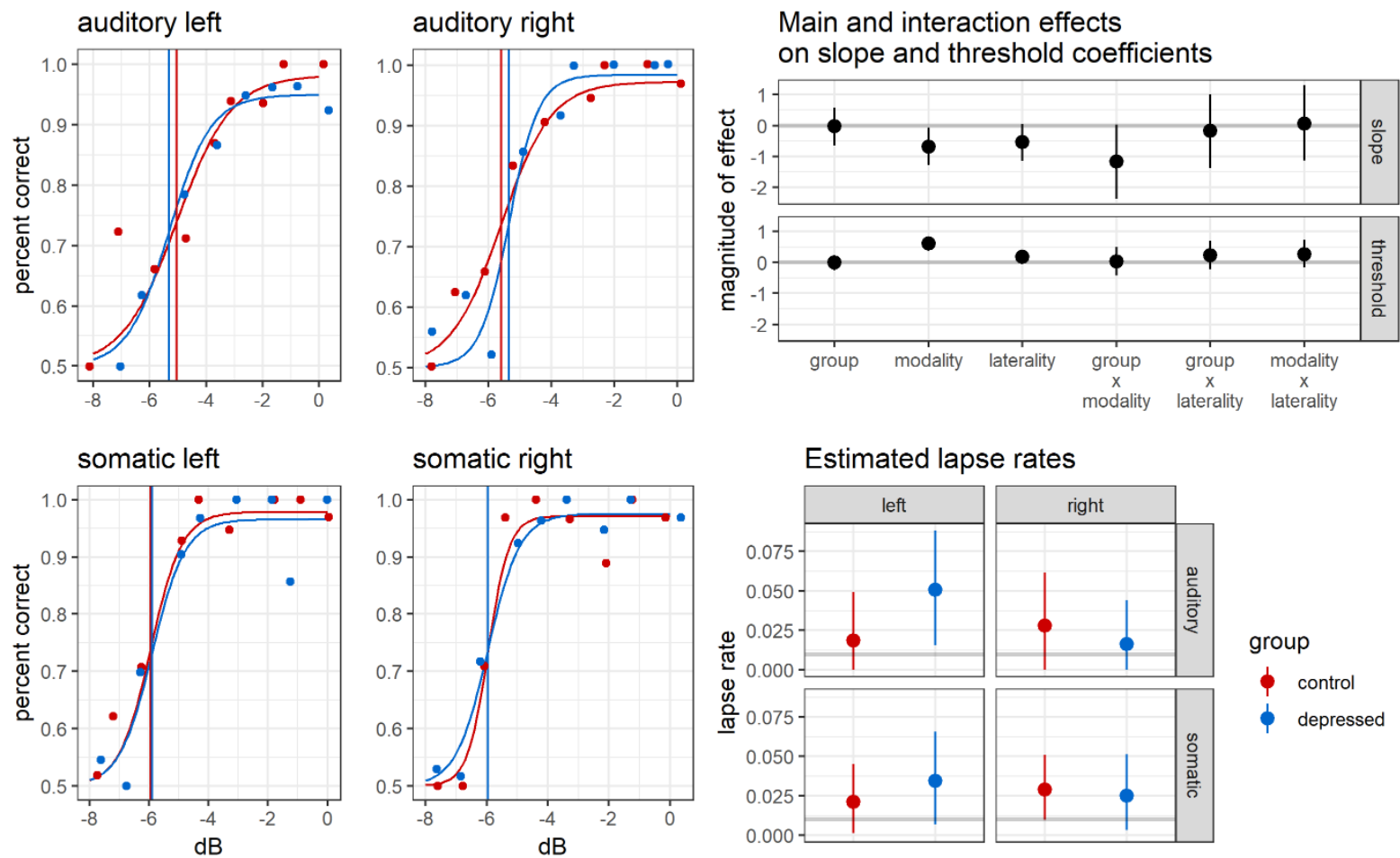

Figure S1. Study 1 GLMM outcomes: medication-free subsample. Left panels: mean logistic curves and thresholds (vertical lines) shown by stimulus modality and laterality. Points represent the observed data (proportion correct averaged over group) shown to the nearest step and jittered to 
avoid overlap. The step size for the auditory staircases is $4 \mathrm{~dB}$, for the somatic staircases $1 \mathrm{~dB}$. Upper right panel: contrasts on effects and interactions for the slope and threshold parameters. Lower right panel: estimated lapse rates by group and condition.

Table S1: Posterior probabilities and Bayes factors for the Study 1 variable selection procedure (medication-free subsample)

\begin{tabular}{|c|c|c|c|}
\hline Model & Effects included & Posterior model & Bayes factor \\
& & probability & relative to \\
& & & reference model \\
\hline $\mathrm{M}$ & Modality & 0.99992 & $6.68 \times 10^{9}$ \\
\hline $\mathrm{ML}$ & Modality and laterality & $5.70 \times 10^{-5}$ & $3.80 \times 10^{5}$ \\
\hline $\mathrm{GM}$ & Group and modality & $2.12 \times 10^{-5}$ & $1.42 \times 10^{5}$ \\
\hline $\mathrm{REF}$ & None (reference model) & $1.50 \times 10^{-10}$ & 1 \\
\hline $\mathrm{L}$ & Laterality & $4.32 \times 10^{-12}$ & $2.89 \times 10^{-2}$ \\
\hline $\mathrm{G}$ & Group & $6.62 \times 10^{-13}$ & $4.42 \times 10^{-3}$ \\
\hline $\mathrm{GML}$ & Group, modality and laterality & $9.35 \times 10^{-14}$ & $6.25 \times 10^{-4}$ \\
\hline $\mathrm{GL}$ & Group and laterality & $9.04 \times 10^{-17}$ & $6.04 \times 10^{-7}$ \\
\hline
\end{tabular}

Study 2 hierarchical signal detection model. The signal detection analysis described in Study 2 was repeated including $\mathrm{n}=6$ unmedicated depressed participants and $\mathrm{n}=12$ healthy controls. Outcomes with respect to bias did not substantively differ from the whole-group analyses described in the main study (Figure S2), although the interaction between group and intensity, which was marginal in the main analysis, became credible in the medication-free analysis. This outcome demonstrates that bias effects are strongest when uncertainty is high (i.e., at low intensity levels) as 
expected. With respect to sensitivity, a credible effect of group on d' emerged, indicating that healthy controls had slightly higher perceptual sensitivity than unmedicated depressed participants. However, the variable selection procedure (Table S2) demonstrated that no group difference in $\mathrm{d}^{\prime}$ remained the most probable explanation for this data, although the Bayes Factor is reduced relative to the wholegroup analysis. It is also worth noting that bias affects sensitivity and the marked bias observed in these data indicates that sensitivity estimates should be treated with caution. Note that the relative likelihood of group differences in bias is substantially increased in the medication-free analysis compared to to the main analysis.

$$
\text { group } \square \text { control } \square \text { depressed }
$$

joint posterior distribution of $d^{\prime}$ and $c$

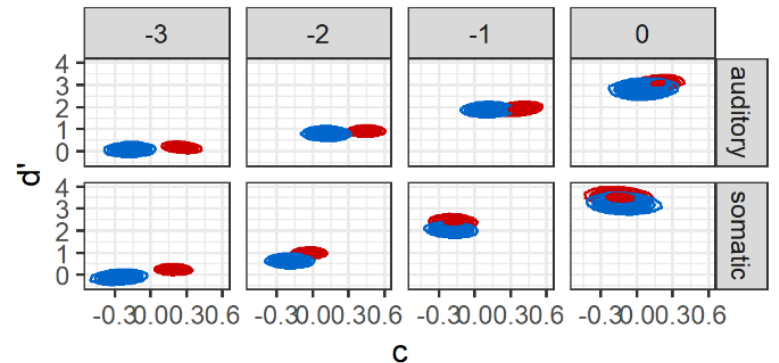

d' by stimulus intensity

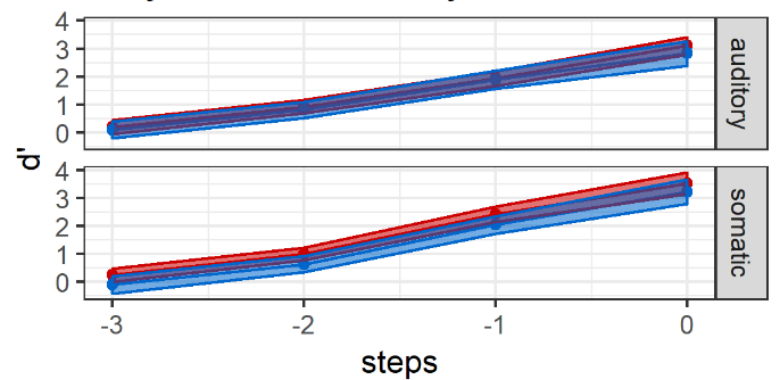

Main and interaction effects on d' and

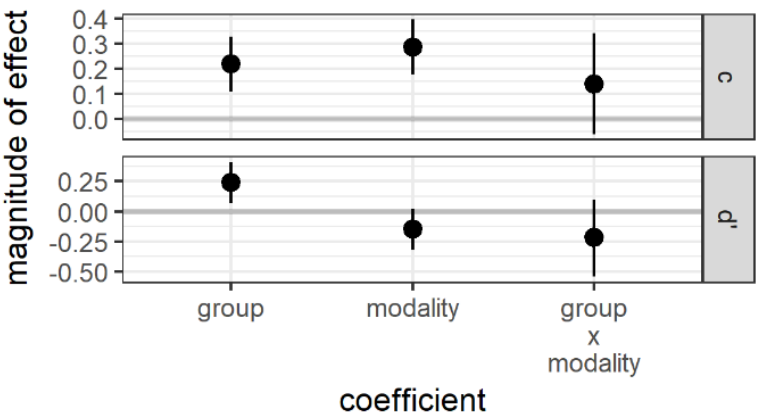

1605

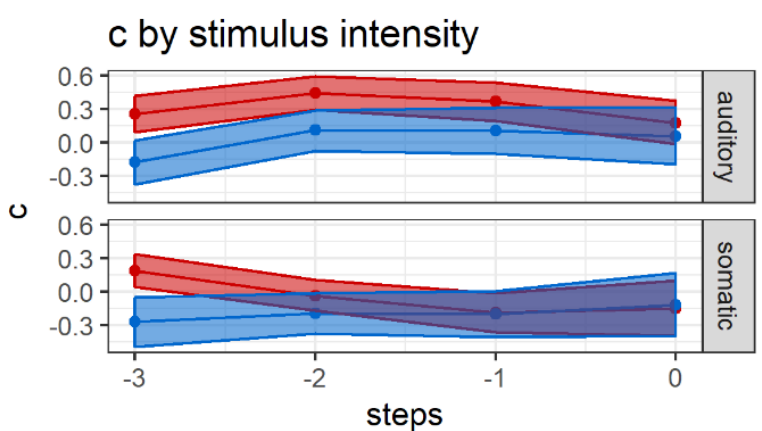

individual c scores at high uncertainty

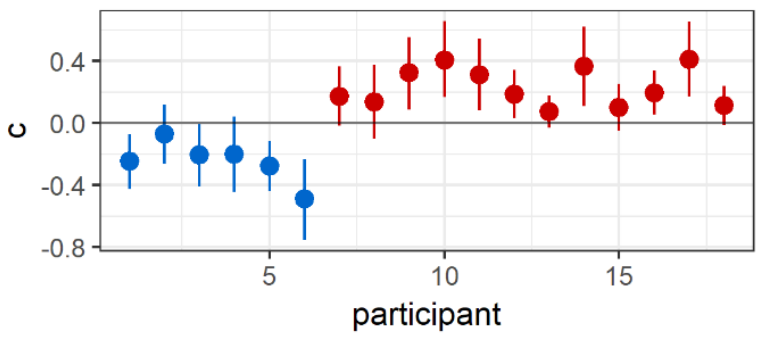

Linear trend analyses on d' and c

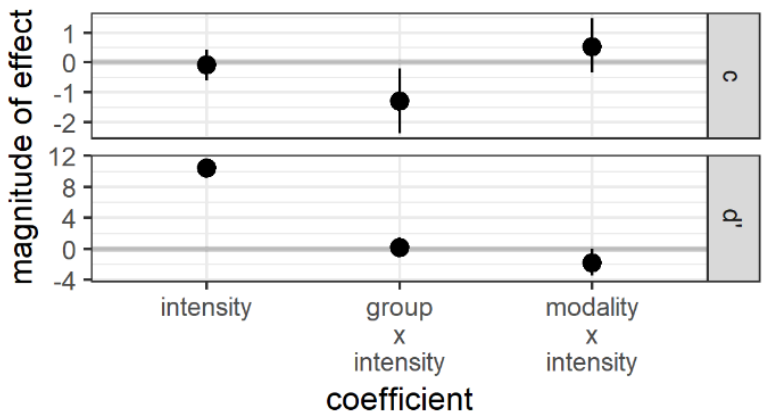


Figure S2: Hierarchical signal detection model outcomes for the medication-free subsample. The top left panel shows the joint posterior distribution of $d^{\prime}$ and $c$ across stimulus intensity, averaged by group and modality, while the middle left and upper right panels respectively show the corresponding $95 \%$ credible intervals for the averaged estimates of $d^{\prime}$ and $c$ across stimulus intensity. The middle right panel shows the individual participant estimates of $\mathrm{c}$ averaged over modality at the lowest level of stimulus intensity. The bottom panels show the main and interaction effects of the linear models on $d^{\prime}$ and $c$ : in the bottom left panel simple contrasts on factors with 2 levels; in the bottom right panel linear trends on intensity and on the respective interactions of group and modality with intensity.

Table S2: Posterior probabilities and Bayes factors for signal detection model comparison (medication-free sample)

\begin{tabular}{|c|c|c|c|c|}
\hline \multirow{2}{*}{ Model } & Model & \multicolumn{2}{|c|}{ Posterior model probability } & Bayes factor \\
\hline \multirow{2}{*}{$d^{\prime}$} & & \multicolumn{2}{|c|}{ c } & \\
\hline & Group effect & 0.29 & 0.004 & 77.58 \\
\cline { 2 - 5 } & No group effect & 0.70 & 0.009 & 77.31 \\
\hline Bayes factor & & 0.41 & 0.41 & \\
\hline
\end{tabular}

Section 2: Study 2 distractor word lists

Table S3: Distractor word lists

\begin{tabular}{|l|l|l|}
\hline Negative words & Positive words & Neutral words \\
\hline Worthless & Valued & Elastic \\
\hline Useless & Effective & Definite \\
\hline Inadequate & Confident & Fluid \\
\hline
\end{tabular}




\begin{tabular}{|c|c|c|}
\hline Contempt & Compassion & Observation \\
\hline Disapproval & Approval & Viewpoint \\
\hline Disparagement & Tenderness & Journey \\
\hline Ridicule & Appreciation & Temperature \\
\hline Blame & Acceptance & Notification \\
\hline Criticize & Praise & Discuss \\
\hline Bad & Welcome & Tiled \\
\hline Fault & Achievement & Arrival \\
\hline Mistake & Skill & Episode \\
\hline Wrong & Justified & Audible \\
\hline Lazy & Active & Solid \\
\hline Selfish & Generous & Rapid \\
\hline Hopeless & Hopeful & Woven \\
\hline Despair & Joy & Motion \\
\hline Desperate & Relaxed & Varnished \\
\hline Trapped & Independent & Layered \\
\hline Stuck & Calm & Short \\
\hline Helpless & Influential & Ductile \\
\hline Burden & Benefit & Demonstration \\
\hline Affliction & Blessing & Postponement \\
\hline Broken & Fulfilled & Narrow \\
\hline Ruined & Lively & Wide \\
\hline Frustration & Satisfaction & Volume \\
\hline Anger & Excitement & Compartment \\
\hline
\end{tabular}




\begin{tabular}{|c|c|c|}
\hline Resentment & Anticipation & Domain \\
\hline Sadness & Happiness & Topic \\
\hline Guilt & Innocence & Discourse \\
\hline Shame & Respect & Measurement \\
\hline Humiliation & Dignity & Length \\
\hline Pain & Peace & Width \\
\hline Suffering & Comfort & Fastening \\
\hline Misery & Cheerfulness & Consistency \\
\hline Distress & Pleasure & Mixture \\
\hline Loneliness & Closeness & Magnitude \\
\hline Alone & Popular & Habitual \\
\hline Failure & Success & Portion \\
\hline Disappoint & Delight & Indicate \\
\hline Bleak & Warm & Broad \\
\hline Relentless & Gentle & Unofficial \\
\hline Rubbish & Treasure & Carpet \\
\hline Complain & Soothe & Arrange \\
\hline Dislike & Cherish & Lift \\
\hline Rejected & Loved & Sent \\
\hline Incompetent & Competent & Glossy \\
\hline Incapable & Capable & Regular \\
\hline Loss & Gain & Pause \\
\hline Regret & Contentment & Passage \\
\hline Exhausted & Energetic & Oval \\
\hline
\end{tabular}




\begin{tabular}{|c|c|c|}
\hline Defeat & Victory & Arrangement \\
\hline Beaten & Proud & Occasional \\
\hline Vulnerable & Strong & Informal \\
\hline Betrayed & Nurtured & Brought \\
\hline Threat & Safety & Texture \\
\hline Powerless & Powerful & Formal \\
\hline Miserable & Fun & Proximal \\
\hline Boring & Interesting & Brief \\
\hline Unpleasant & Pleasant & Extended \\
\hline Fail & Succeed & Proceed \\
\hline Isolated & Supported & Viewed \\
\hline Paralysing & Exhilarating & Entering \\
\hline Illness & Health & Sequence \\
\hline Wounded & Flourishing & Moist \\
\hline Injured & Robust & Dry \\
\hline Ailment & Vigour & Compartment \\
\hline Poison & Wellness & Angle \\
\hline Sick & Fit & Rectangular \\
\hline Infected & Healthy & Folded \\
\hline Weakness & Strength & Velocity \\
\hline Disgusting & Attractive & Medium \\
\hline Polluted & Pure & Plastic \\
\hline Diseased & Thriving & Scanned \\
\hline Dreary & Inspiring & Neutral \\
\hline
\end{tabular}




\begin{tabular}{|l|l|l|}
\hline Dull & Vibrant & Substantial \\
\hline Aching & Comfortable & Stationary \\
\hline Hurt & Healed & Borrowed \\
\hline Pathetic & Admirable & Ajar \\
\hline Stupid & Bright & Powdery \\
\hline Harm & Kindness & Situation \\
\hline Contaminated & Fresh & Spatial \\
\hline
\end{tabular}

1621

1622

1623

1624

1625

1626

1627

1628

Linear regression. According to the model, the (standardised) data is distributed as

$$
y_{i} \sim N\left(\mu, \sigma^{2}\right)
$$

$$
\begin{gathered}
\mu=\beta 0+\beta 1 x_{i} \\
\sigma \sim \operatorname{Cauchy}(0,2), \sigma \geq 0
\end{gathered}
$$




$$
\begin{aligned}
& \beta 0 \sim N\left(0, \sigma^{\beta 0^{2}}\right) \\
& \beta 1 \sim N\left(0, \sigma^{\beta 1^{2}}\right) \\
& \sigma^{\beta 0}=\sigma^{\beta 1}=2
\end{aligned}
$$

As expected, there was a credible association between anhedonia and depression severity in both studies. A trend towards an association between left-sided response bias and depression severity was not credibly different from zero (Figure S3, lower panels). Residuals were calculated for each regression and a point estimate for each residual derived from the mean of the posterior distribution for that residual.

Poisson fit to lapse data distribution

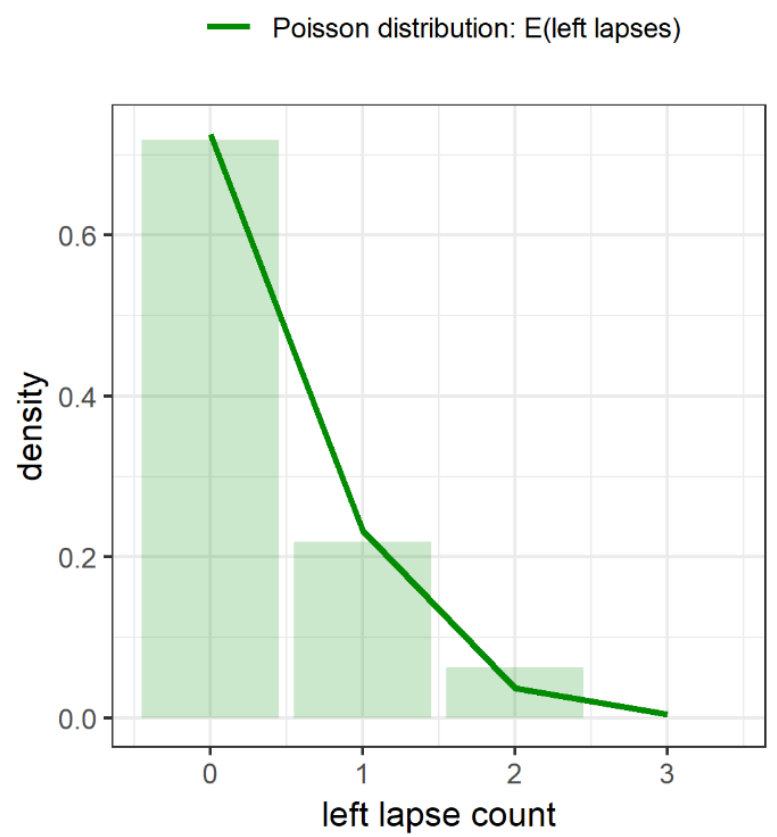

Scatterplot of left lapses against BDI

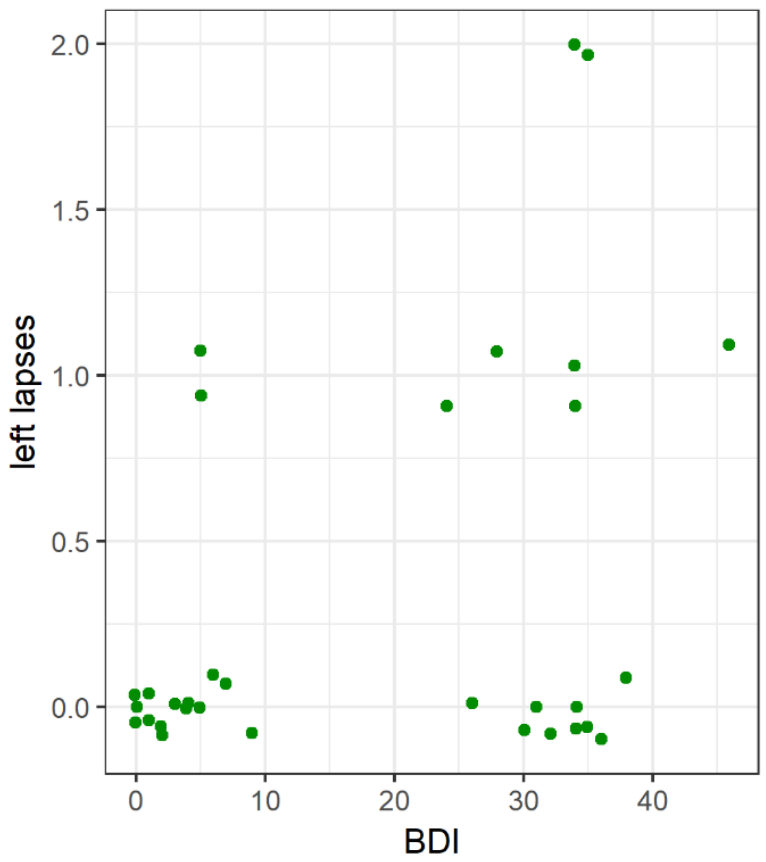

Posterior probabilities for regression coefficients
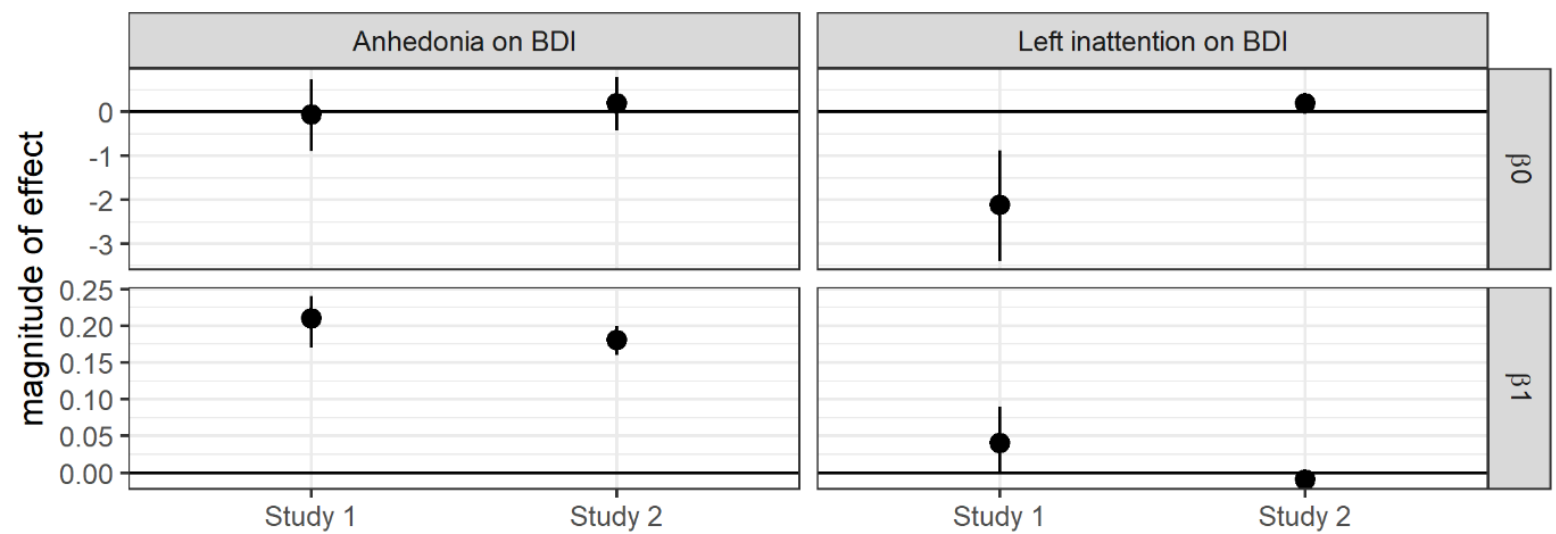
Figure S3: Linear and Poisson regression indices. Upper left panel: Study 1 Poisson distribution fit to left lapse count data. Upper right panel: Study 1 scatterplot of left lapses against total BDI score (jittered). Lower panels: posterior probabilities for the coefficients for intercept $(\beta 0)$ and slope $(\beta 1)$ for each regression. There was a credible association between anhedonia and BDI score in both studies. In both studies there was a trend towards a relationship between total BDI score and studyspecific indices of lateralised attentional bias, but in neither study was it credibly different from zero.

Poisson regression. Given that the left-sided lapse variable is a count variable, the data was informally examined for fit to a Poisson distribution. A single outlier was 6.14 standards deviations from the mean of the distribution of all other counts, and 5.61 standard deviations from its predicted value given the regression model. The remaining data showed little evidence of overdispersion (Figure S3, upper panels).

The left-sided lapses were modelled as draws from a Poisson distribution

$$
y_{i} \sim \operatorname{Poisson}\left(\lambda_{i}\right)
$$

where $y_{i}$ denotes the outcome for the $\mathrm{i}^{\text {th }}$ participant and where

$$
\begin{gathered}
\log \left(\lambda_{i}\right)=\beta 0+\beta 1 x_{i} \\
\beta 0 \sim N\left(0, \sigma^{\beta 0^{2}}\right) \\
\beta 1 \sim N\left(0, \sigma^{\beta 1^{2}}\right) \\
\sigma^{\beta 0}=\sigma^{\beta 1}=2
\end{gathered}
$$

There was a trend towards an association between left-sided lapses and depression severity (Figure S3, lower right panel). As before, residuals were calculated for the regression and a point estimate for each residual derived from the mean of the posterior distribution for that residual. 
1661 Partial correlations. A coefficient indexing the partial correlation between anhedonia and 1662 attentional bias, controlling for depression severity, was then estimated for each study. For each 1663 study, the residuals were modelled as draws from a multivariate normal distribution as follows 1664 (adapted from [33])

$$
\text { Residuals }_{\boldsymbol{i}} \sim N\left(\left[\mu_{1}, \mu_{2}\right],\left[\begin{array}{cc}
\sigma_{1}^{2} & r \sigma_{1} \sigma_{2} \\
r \sigma_{1} \sigma_{2} & \sigma_{2}^{2}
\end{array}\right]\right)
$$

1665 where

$$
\begin{gathered}
\mu_{1}, \mu_{2} \sim N(0,100) \\
r \sim \operatorname{Uniform}(0,1) \\
\sigma_{1}, \sigma_{2} \sim \operatorname{Cauchy}(0,5), \sigma \geq 0
\end{gathered}
$$

1666 The distributions for the partial correlation coefficients were credibly different from zero for each 1667 study: in Study 1 the correlation coefficient had a mean magnitude of 0.40 and lower and upper HDI 1668 limits of 0.11 and 0.66 ; while in Study 2 the coefficient had a mean magnitude of -.57 and lower and 1669 upper HDI limits of -0.29 and -0.82 . Note that this correlation is negative because left-sided bias was 1670 denoted by negative values for c: in both studies these outcomes demonstrate that a higher 1671 anhedonia score is credibly related to more evidence of left-sided attentional bias. 